\title{
Enhanced Activation of HCN Channels Reduces Excitability and Spike-Timing Regularity in Maturing Vestibular Afferent Neurons
}

\author{
Christopher M. Ventura ${ }^{1}$ and $\odot$ Radha Kalluri ${ }^{1,2,3}$ \\ ${ }^{1}$ Neuroscience Graduate Program, ${ }^{2}$ Department of Otolaryngology-Head and Neck Surgery, Keck School of Medicine, and ${ }^{3}$ Zilkha Neurogenetic Institute, \\ Keck School of Medicine, University of Southern California, Los Angeles, California, 90057
}

Vestibular ganglion neurons (VGNs) transmit information along parallel neuronal pathways whose signature distinction is variability in spike-timing; some fire at regular intervals while others fire at irregular intervals. The mechanisms driving timing differences are not fully understood but two opposing (but not mutually exclusive) hypotheses have emerged. In the first, regular-spiking is inversely correlated to the density of low-voltage-gated potassium currents $\left(I_{\mathrm{KL}}\right)$. In the second, regular spiking is directly correlated to the density of hyperpolarization-activated cyclic nucleotide-sensitive currents $\left(I_{\mathrm{H}}\right)$. Supporting the idea that variations in ion channel composition shape spike-timing, VGNs from the first postnatal week respond to synaptic-noise-like current injections with irregular-firing patterns if they have $I_{\mathrm{KL}}$ and with more regular firing patterns if they do not. However, in vitro firing patterns are not as regular as those in vivo. Here we considered whether highly-regular spiking requires $I_{\mathrm{H}}$ currents and whether this dependence emerges later in development after channel expression matures. We recorded from rat VGN somata of either sex aged postnatal day (P)9-P21. Counter to expectation, in vitro firing patterns were less diverse, more transient-spiking, and more irregular at older ages than at younger ages. Resting potentials hyperpolarized and resting conductance increased, consistent with developmental upregulation of $I_{\mathrm{KL}}$. Activation of $I_{\mathrm{H}}$ (by increasing intracellular cAMP) increased spike rates but not spike-timing regularity. In a model, we found that activating $I_{\mathrm{H}}$ counter-intuitively suppressed regularity by recruiting $I_{\mathrm{KL}}$. Developmental upregulation in $I_{\mathrm{KL}}$ appears to overwhelm $I_{\mathrm{H}}$. These results counter previous hypotheses about how $I_{\mathrm{H}}$ shapes vestibular afferent responses.

Key words: cAMP; $I_{\mathrm{H}} ; I_{\mathrm{KL}}$; neuronal excitability; spike-timing regularity; vestibular ganglion

Significance Statement

Vestibular sensory information is conveyed on parallel neuronal pathways with irregularly-firing neurons encoding information using a temporal code and regularly-firing neurons using a rate code. This is a striking example of spike-timing statistics influencing information coding. Previous studies from immature vestibular ganglion neurons (VGNs) identified hyperpolarizationactivated mixed cationic currents $\left(I_{\mathrm{H}}\right)$ as driving highly-regular spiking and proposed that this influence grows with the current during maturation. We found that $I_{\mathrm{H}}$ becomes less influential, likely because maturing VGNs also acquire low-voltage-gated potassium currents $\left(I_{\mathrm{KL}}\right)$, whose inhibitory influence opposes $I_{\mathrm{H}}$. Because efferent activity can partly close $I_{\mathrm{KL}}$, VGN firing patterns may become more receptive to extrinsic control. Spike-timing regularity likely relies on dynamic ion channel properties and complementary specializations in synaptic connectivity.

\section{Introduction}

The vestibular system sends information from the sensory periphery to the brainstem along parallel neuronal pathways whose

Received July 13, 2018; revised Jan. 14, 2019; accepted Jan. 19, 2019.

Author contributions: C.M.V. wrote the first draft of the paper; C.M.V. and R.K. edited the paper; C.M.V. and R.K. designed research; C.M.V. performed research; C.M.V. and R.K. analyzed data; C.M.V. and R.K. wrote the paper.

This work was supported by NIH NIDCD Grants R03 DC012652, DC012652S, and R01 DC015512, and the Department of Otolaryngology at the University of Southern California. We thank Maya Monges-Hernandez for technical assistance, and Alexander Markowitz and Ruth Anne Eatock for commenting on previous versions of the paper.

The authors declare no competing financial interests. signature distinction is a striking difference in spike-timing statistics. Spikes occur at regular intervals in one path and at irregular (noisy) intervals in the other (Baird et al., 1988; for review, see Goldberg, 2000). Spike-timing regularity correlates with other important features of the neuronal response, such as gain, phase, and sensitivities to different frequencies of head motion

Correspondence should be addressed to Radha Kalluri at radha@usc.edu.

https://doi.org/10.1523/JNEUROSCI.1811-18.2019

Copyright $\odot 2019$ the authors 
(for review, see Goldberg, 2000). It also appears to determine whether these neurons use a rate or temporal code to represent sensory information (Sadeghi et al., 2007). Given its central importance to vestibular function, the aim of our study is to understand the biophysical mechanisms that drive spike-timing regularity.

Pioneering studies considered many mechanisms to explain spike-timing regularity. These included the type and numbers of sensory hair cells contacted by individual neurons, differences in synaptic and/or dendritic morphology, and regional variations in ion channel composition (Smith and Goldberg, 1986; Baird et al., 1988; Fernández et al., 1988, 1990). Ultimately, these studies concluded that variations in ion channel composition are critical for driving differences in spike-timing regularity (Highstein and Politoff, 1978; Smith and Goldberg, 1986; Schessel et al., 1991), but the exact ion channel substrate remains under debate.

Electrophysiological and immunohistochemical data in rats and mice have shown expression for many groups of ion channels, both in the terminals and at the somata of vestibular afferents (Desmadryl et al., 1997; Chabbert et al., 2001; Risner and Holt, 2006; Iwasaki et al., 2008; Kalluri et al., 2010; Lysakowski et al., 2011; Almanza et al., 2012; Horwitz et al., 2014; Yoshimoto et al., 2015). For example, potassium channel expression varies by epithelial zone and could drive zonal variations in spike-timing regularity (Songer and Eatock, 2013; Meredith and Rennie, 2015). Indicative of heterogeneity in ion channel composition, vestibular ganglion neuron (VGN) somata respond with diverse firing patterns when injected with step currents. Somata with large low voltage-gated potassium currents $\left(I_{\mathrm{KL}}\right)$ respond only at the onset of the current step (transient/phasic), whereas those with little $I_{\mathrm{KL}}$ respond with sustained spike trains (sustained/ tonic; Fig. 1). When driven with currents that mimic the random timing of synaptic input, transient-spiking VGNs respond with irregularly-timed spike trains, whereas sustained-spiking VGNs respond with more regular patterns (Kalluri et al., 2010). Although these results suggest that $I_{\mathrm{KL}}$ can control spike-timing regularity, in vitro firing patterns do not have the degree of regularity seen in vivo.

Recent studies suggest that highly-regular spiking relies on the depolarizing currents provided by the hyperpolarizationactivated cyclic nucleotide ( $\mathrm{HCN}$ )-sensitive currents (i.e., $I_{\mathrm{H}}$ currents conducted by HCN channels; Horwitz et al., 2014; Yoshimoto et al., 2015). This hypothesis is appealing because $I_{\mathrm{H}}$ produces highly regular auto-rhythmic firing in other systems, famously in sinoatrial node myocytes allowing for pacemaking in the heart (Brown et al., 1979; DiFrancesco, 1981, 2010). Because $I_{\mathrm{H}}$ expression in VGNs grows during development (Yoshimoto et al., 2015) and the current is sensitive to cyclic-nucleotide concentrations (for review, see DiFrancesco, 2010; for VGNs, see Almanza et al., 2012), its influence on spiking could be masked by experimental conditions.

To understand the ion channel substrate necessary for driving variations in spike-timing regularity, we performed in vitro whole-cell patch-clamp on isolated VGNs at ages when HCN channel expression has matured and in experimental conditions where the activation of the $I_{\mathrm{H}}$ current is enhanced by increasing intracellular concentrations of cAMP. We report that enhancing $I_{\mathrm{H}}$ increased spike rate, but failed to produce highly regular firing. Using a single compartment model of VGNs, we show that opposing interactions between $I_{\mathrm{H}}$ and $I_{\mathrm{KL}}$ currents can explain the lack of highly regular firing.

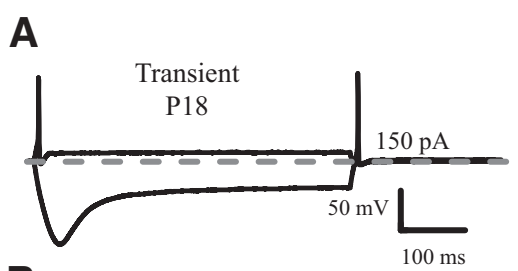

B

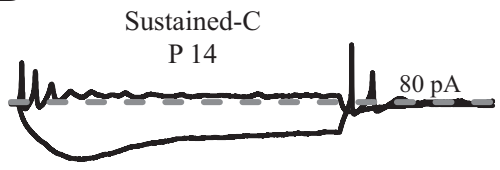

C

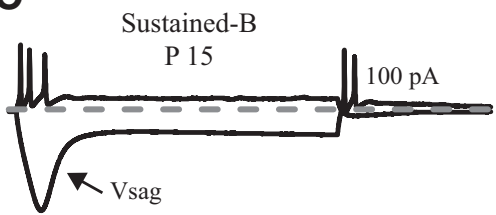

D

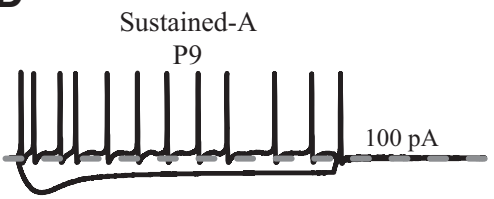

E

Sustained-A $35^{\circ} \mathrm{C}$

P12

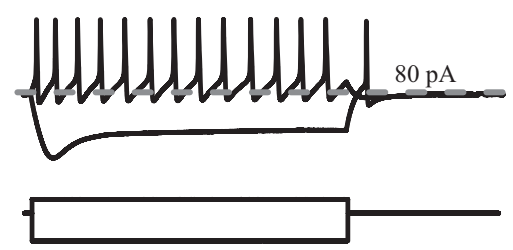

Figure 1. Vestibular ganglion neurons produce different firing patterns in response to depolarizing current steps. $A$, Transient-firing neurons are characterized by a single action potential at the onset of suprathreshold depolarizing steps. $\boldsymbol{B}$ - $\boldsymbol{D}$, Sustained-firing patterns are more variable. $\boldsymbol{B}$, Sustained-C neurons have one or two spikes followed by prominent voltage oscillations. C, Sustained-B neurons show adaptation, producing several spikes at current onset and then cease firing (accommodate). $D$, Sustained-A neurons produce long spike trains throughout the current step. $\boldsymbol{E}$, Sustained-A neuron at $35-37^{\circ} \mathrm{C}$ in $500 \mu \mathrm{m} \mathrm{db}$-CAMP, which produces more spikes than in sustained-A neuron shown in $\boldsymbol{D}$. The amplitude of the depolarizing current step that evokes spikes (current threshold) is shown in the label at the right of each trace and the dashed line represents $-60 \mathrm{mV}$. Indicative of the presence of hyperpolarization-activated mixed cationic current $\left(I_{H}\right)$, most $V G N$ s produced a voltage sag (arrow labeled $V_{\text {sag }}$ ) in response to large hyperpolarizing currents ( $-200 \mathrm{pA}$ shown). Scale bars (top right trace) apply to all traces. For this figure, recordings were made using whole-cell ruptured-patch with $100 \mu \mathrm{m}$ cAMP, except where the recording pipette contained $500 \mu \mathrm{m} \mathrm{db}$-CAMP.

\section{Materials and Methods}

\section{Preparations}

Data were recorded from the superior portion of the vestibular ganglion in Long-Evans rats of either sex aged postnatal day (P)9-21 (P0, birth day). All animals were handled and housed in accordance with National Institutes of Health Guide for the Care and Use of Laboratory Animals. All animal procedures were approved by the University of Southern California Institutional Animal Care and Use Committee. Chemicals were obtained from Sigma-Aldrich unless otherwise specified. The temporal bones from the animals were dissected in chilled and oxygenated L-15 supplemented with $10 \mathrm{~mm}$ HEPES (Leibowitz medium). The superior part of the vestibular ganglia was detached from the distal and central nerve branches freeing it from the otic capsule. Bone fragments, debris, and any remaining connective tissue were removed from the surface of 
the ganglia. Ganglia from 2 to 4 litter-matched animals of either sex were pooled together. Ganglionic tissue was then incubated at $37^{\circ} \mathrm{C}$ in a solution of L-15 with $0.05 \%$ collagenase and $0.25 \%$ trypsin for $20-45 \mathrm{~min}$, depending on age of the animal. The ganglia were then washed in fresh L-15 solution, dissociated by triturating through a series of polished Pasteur pipettes and allowed to settle onto poly-D-lysine-coated glass bottom culture dishes (MatTek). Culture dishes contained bicarbonatebuffered culture medium (minimal essential medium, Invitrogen), supplemented with $10 \mathrm{~mm}$ HEPES, 5\% FBS, and $1 \%$ penicillin-streptomycin (Invitrogen). Cells were incubated for $16-24 \mathrm{~h}$ in $5 \% \mathrm{CO}_{2} / 95 \%$ air at $37^{\circ} \mathrm{C}$. Short-term incubation tends to remove supporting and satellite cells, debris from enzyme treatment, and promotes successful recordings as the animals entered the second postnatal week and beyond.

\section{Electrophysiology}

Cells were viewed at $400 \times$ using an inverted microscope (Zeiss, Axiovert 135 TV) fitted with Nomarski optics. A MultiClamp 700B amplifier, Digidata 1440 board, and pClamp 10.7 software (MDS; RRID: SCR_011323) were used to deliver, record, and amplify all signals. Custom stimuli were generated in MATLAB (MathWorks; RRID: SCR_001622) and delivered via the pClamp software. Recording pipettes were fabricated using filamented borosilicate glass. Pipettes were fire polished to yield an access resistance between 4 and $8 \mathrm{M} \Omega$. Recording pipettes were coated with a silicone elastomer (Sylgard 184; Dow Corning) to reduce pipette capacitance.

The properties of ion channels were studied using whole-cell ruptured-patch or perforated-patch methods. The contents of the standard internal solution contained the following (in $\mathrm{mM}$ ): $135 \mathrm{KCl}, 3.5$ $\mathrm{MgCl}_{2}, 3 \mathrm{Na}_{2} \mathrm{ATP}, 5 \mathrm{HEPES}, 5$ EGTA, $0.1 \mathrm{CaCl}_{2}, 0.1 \mathrm{Li}-\mathrm{GTP}$, and titrated with $1 \mathrm{M} \mathrm{KOH}$ to a $\mathrm{pH}$ of 7.35 and an osmolarity of $\sim 300 \mathrm{mmol} / \mathrm{kg}$. Both cAMP and dibutyryl (db)-cAMP were aliquoted and stored as a $10 \mathrm{~mm}$ stock at $-20^{\circ} \mathrm{C}$. Working concentrations were achieved by dilution into the intracellular solution on day of recording. Endogenous conditions were studied using the perforated patch-clamp method where the intracellular milieu is relatively unchanged. The contents of the perforatedpatch internal solution contained the following (in $\mathrm{mM}$ ): $75 \mathrm{~K}_{2} \mathrm{SO}_{4}, 25$ $\mathrm{KCl}, 5 \mathrm{MgCl}_{2}, 5 \mathrm{HEPES}, 5$ EGTA, $0.1 \mathrm{CaCl}_{2}$, and titrated with $1 \mathrm{M} \mathrm{KOH}$ to a $\mathrm{pH}$ of 7.4. Amphotericin B (240/ml; Sigma-Aldrich) was dissolved in DMSO and added to the perforated-patch solution on day of recording. This allowed passage of small monovalent ions while preventing larger molecules from dialyzing.

The series resistance ranged between 8 and $35 \mathrm{M} \Omega$ (higher values for perforated patch) and was compensated by 70 and $50 \%$, respectively; giving final resistances between 2.5 and $17 \mathrm{M} \Omega$. Uncompensated resistance was corrected off-line. Recordings were made at room temperature $\left(25-27^{\circ} \mathrm{C}\right)$, unless otherwise stated, and in an external bath continuously perfused with fresh oxygenated L-15 media. Standard internal solution and perforated patch internal solution had junction potentials of +4.3 and $+5.0 \mathrm{mV}$, respectively. In some experiments the bath was heated to between $35^{\circ} \mathrm{C}$ and $37^{\circ} \mathrm{C}$ with a heated platform and temperature controller (TC-344B; Warner Instruments). To maintain bath temperature with stability, room humidity was maintained using a bench-top humidifier. Junction potentials were computed with JPCalc (Barry, 1994) as implemented in pClamp 10.7 and not corrected for. Only recordings in which the cell had formed a gigaohm seal and maintained stable membrane potential were used. The stability of neurons was monitored by measuring resting potential, input resistance, firing patterns, and in some neurons the activation properties of $I_{\mathrm{H}}$, at regular intervals throughout the recording period.

\section{Pharmacology}

$\mathrm{I}_{H}$ antagonists. A stock solution of ZD7288 was stored at $-20^{\circ} \mathrm{C}$ in $10 \mathrm{mM}$ aliquots. An $80 \mu \mathrm{M}$ working concentration was achieved by dilution in either L-15 for extracellular administration or in standard internal solution for intracellular administration. A $2 \mathrm{~mm}$ stock solution of $\mathrm{CsCl}$ in L-15 was prepared fresh on the day of recording for extracellular administration. Drugs were applied via a pressurized super-perfusion system (Warner Instruments). Measurements of $I_{\mathrm{H}}$ were made at least 2-5 min after exposure to the drug.
A

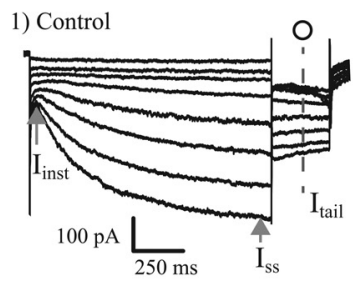

2) $2 \mathrm{mM} \mathrm{CsCl}$

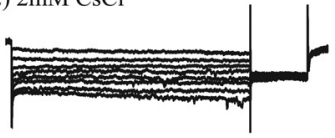

3) 1 - 2
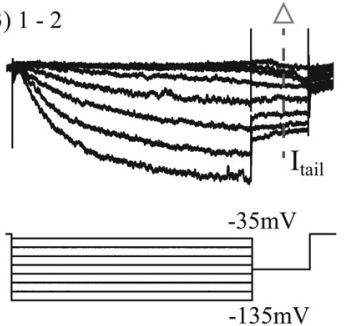

B

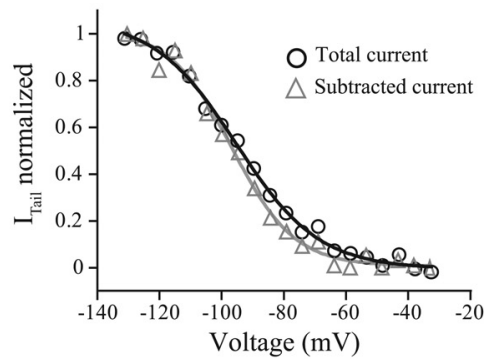

Figure 2. An electrophysiological and pharmacological method for characterizing the activation properties of $I_{\mathrm{H}}$. Current excited by a family of long duration $(1.7 \mathrm{~s}$ ) conditioning voltage steps ranging from -135 to $-35 \mathrm{mV}$ in $5 \mathrm{mV}$ increments followed by a 300 ms tail step to $-100 \mathrm{mV}$. For clarity, not every voltage-step response is shown. Current under control conditions (A1) and with $\mathrm{CsCl}(\boldsymbol{A 2})$ and the $\mathrm{CS}^{+}$-sensitive current obtained by subtraction 1-2 (A3). Gray arrows, The amplitude of the $I_{H}$ current was determined by taking the difference between the $I_{\text {inst }}$ and the $I_{S S}$. $B$, The voltage-dependent activation of $I_{H}$ is determined by plotting the magnitude of the tail current $\left(I_{\text {tail }}\right)$ as a function of the conditioning voltage. Curves were fit by a Boltzmann function and normalized by the maximum current. The fits generated from the tail of the control condition $(\bigcirc)$ and from the current isolated from the $C s+$ induced block $(\Delta)$ are similar. All voltage-dependent characterization of $I_{H}$ in subsequent figures is derived from the control condition.

\section{Analysis}

General. All data were analyzed with pClamp 10 software (Clampfit; MDS Analytical Technologies). Input resistance $\left(R_{\text {in }}\right)$ was calculated from voltage changes in response to a hyperpolarizing 10 or $20 \mathrm{pA}$ step of current. The membrane time constant $\left(\tau_{\mathrm{m}}\right)$ was acquired by fitting the resulting voltage response with a single exponential. Membrane capacitance $\left(C_{\mathrm{m}}\right)$ was computed as the ratio of membrane time constant and input resistance $\left(\tau_{\mathrm{m}} / R_{\mathrm{in}}\right)$.

Statistical analysis was done with Origin Pro (OriginLab; RRID: SCR_014212) and/or JMP Pro 13 (SAS Institute; RRID:SCR_014242). Statistical significance was estimated with Student's $t$ test when variances were equal or with Welch's $t$ test when variances were not equal. We used an $\alpha$ level of 0.05 for all statistical tests. One-way and two-way ANOVAs were applied followed by a post hoc Tukey's HSD analysis, as required, to examine the influence of cAMP concentration/condition on $I_{\mathrm{H}}$ half activation, firing rate and coefficient of variation (CV). Statistics are reported as means \pm SEM.

$I_{H}$ activation parameters. We studied the voltage-dependent activation of $I_{\mathrm{H}}$ using a voltage-clamp tail-current protocol (Fig. 2). The HCN 
channel that carries $I_{\mathrm{H}}$ opens very slowly and the voltage-dependent activation of $I_{\mathrm{H}}$ is sensitive to the length of hyperpolarization. We designed a protocol that allowed ample time for the channel to activate and provided an accurate measurement of the voltage-dependent activation. The challenge is that neurons can die when held at hyperpolarized voltages for extended periods of time. The protocol begins with a preconditioning voltage step, then a $-100 \mathrm{mV}$ "tail-step" for $300 \mathrm{~ms}$ and finally returns to $-60 \mathrm{mV}$ for $100 \mathrm{~ms}$. The precondition voltage step is incremented by $5 \mathrm{mV}$ to range between -135 and $-40 \mathrm{mV}$. A $15 \mathrm{~s}$ delay was implemented between each step to allow enough time for HCN channels to close completely before starting the next voltage step. Several preconditioning lengths were tested $(1.25,1.7$, and $2.5 \mathrm{~s})$. Although the $2.5 \mathrm{~s}$ protocol provided the most depolarized measures of voltage-dependent activation and had the most complete $I_{\mathrm{H}}$ activation, we found that $1.7 \mathrm{~s}$ provided comparable results without killing the neuron. The voltagedependent activation of $I_{\mathrm{H}}$ is characterized by plotting the currents flowing during the tail step $\left(I_{\text {tail }}\right)$ against the precondition voltage $\left(V_{\text {pre }}\right)$. These data were well fit with a Boltzmann function (Eq. 1):

$$
I_{\text {tail }}=\frac{I_{\max }}{\left[1+\exp ^{\frac{V_{1 / 2}-V_{p r e}}{k}}\right]}+C
$$

where $I_{\max }$ is the maximum current measured during the tail, $V_{\text {pre }}$ is the preconditioning/test voltage, $V_{1 / 2}$ is the half-activation potential, $k$ is the slope factor, and $C$ is a constant. Measurements of $I_{\text {tail }}$ were taken $100 \mathrm{~ms}$ into the tail step to avoid contamination from other low-voltage-gated currents, particularly during the most positive voltage steps ( -60 to -40 $\mathrm{mV}$ ) and when $I_{\mathrm{H}}$ was activated with cAMP/db-cAMP.

The voltage-dependent time course for $I_{\mathrm{H}}$ was estimated by fitting the current evoked at the $-135 \mathrm{mV}$ preconditioning step with a single exponential. All fits were obtained after a $60 \mathrm{~ms}$ delay from the start of the voltage step. Only fits with a correlation coefficient $\geq 0.985$ were retained. Mean $\tau$ values from the single exponential fit at $-135 \mathrm{mV}$ were $\sim 327.9 \pm 30.7 \mathrm{~ms}(n=20)$, which are consistent with those reported by Almanza et al. (2012) in VGNs. We also tried fitting with the sum of two exponentials, which improved fits for nearly $80 \%$ of cells at $-135 \mathrm{mV}$ (Almanza et al., 2012; Liu et al., 2014), but this type of fit failed more often at depolarized potentials. To yield consistent fits as a function of voltage, we relied on the single exponential fits, which were uniformly good for all cells and voltages.

\section{Pseudo-synaptic stimuli}

To mimic natural synaptic input, we generated trains of simulated EPSCs (pseudo-EPSCs) with pseudorandom timing, as previously described by Kalluri et al. (2010). A stimulus set of 20 pseudo-EPSC sweeps was created and delivered to each VGN in current-clamp mode to drive spiking. Spikes were detected with the built-in spike thresholding algorithms of Clampfit 10.7

For each neuron the $\mathrm{CV}$ gives a measure of the spike-timing regularity. $\mathrm{CV}$ is computed as the SD of the interspike interval (ISI) divided by the mean ISI. Only CV values for neurons in which the SEM spike rate was $<10 \%$ of the mean ISI were included. CV is inherently related to spike rate (Goldberg et al., 1990a). The normalized statistic $\mathrm{CV}^{\star}$ is often used to account for this relationship (Goldberg et al., 1990a). CV* is the CV at a standard spike rate and allows for a measure of regularity independent of spike rate. We did not use $\mathrm{CV}^{\star}$, choosing instead to only compare CVs of neurons that were driven at the same mean spike rate.

Variations in spike rate were achieved by increasing or decreasing the mean amplitude of the pseudo-EPSCs. Although effective in changing spike rate, this is not a completely natural method of stimulation. A more natural stimulus would modulate both EPSC amplitude and EPSC rate. This stimulation method was chosen to maintain timing patterns (by maintaining a standard EPSC rate) to isolate the influence of ion channels from synaptic input.

\section{Vestibular model}

A single compartment conductance-based model was used to test the influence of $I_{\mathrm{H}}$ and $I_{\mathrm{KL}}$ on firing patterns. The model was implemented in
Table 1. Model Parameters for $r_{\infty}$ and $\tau_{\mathrm{r}}$ selected from our experimental data

\begin{tabular}{lccc}
\hline & Min activation, & Max activation, & Heated max-activation, \\
& $100 \mu \mathrm{m}$ cAMP & $500 \mu \mathrm{m} \mathrm{dB}$-cAMP & $500 \mu \mathrm{m} \mathrm{dB}-$ cAMP $+35^{\circ} \mathrm{C}$ \\
\hline $\mathrm{V}_{1 / 2}, \mathrm{mV}$ & 94.11 & 84.37 & 85.38 \\
$\mathrm{k}, \mathrm{slope}$ & 8.1 & 8.6 & 8.8 \\
$\mathrm{a}, \mathrm{mV}$ & 86.862 & 80.646 & 70.911 \\
$\mathrm{~b}, \mathrm{mV}$ & 5.768 & 6.916 & 12.071 \\
$\mathrm{c}$ & 3393.342 & 2551.988 & 1569.342 \\
$\mathrm{~d}, \mathrm{mV}$ & 6.674 & 14.881 & 3.769 \\
$\mathrm{e}$ & 295.958 & 209.479 & 149.331 \\
\hline
\end{tabular}

Table 2. Model parameters

\begin{tabular}{lcll}
\hline & $g_{\max } \mathrm{mS} / \mathrm{cm}^{2}$ & $V_{1 / 2}, \mathrm{mV}$ & Slope \\
\hline$g_{\mathrm{KH}}$ & 2.8 & -15 & 5 \\
gleak & 0.03 & & 8 \\
$g_{\mathrm{KL}}$ & $0-1.1$ & -44.5 & 8.8 \\
$g_{\mathrm{Na}}$ activation & 13 and 20 & -38 & 7 \\
$g_{\mathrm{Na}}$ inactivation & 3393 & -65 & 6 \\
$g_{\mathrm{H}}$ & 0.91 & -84 to -96 & $8.1-8.6$ \\
\hline
\end{tabular}

Table 3. Additional model parameters

\begin{tabular}{lr}
\hline$E_{\mathrm{K}}$ & $-81 \mathrm{mV}$ \\
$E_{\mathrm{Na}}$ & $82 \mathrm{mV}$ \\
$E_{\text {leak }}$ & $-65 \mathrm{mV}$ \\
$E_{\mathrm{H}}$ & $-46 \mathrm{mV}$ \\
$C_{\mathrm{m}}$ & $0.9 \mathrm{pF} / \mathrm{cm}^{2}$ \\
\hline
\end{tabular}

MATLAB as a differential equation in which the net current across the neuronal membrane was taken as the sum of currents flowing through individual circuit elements connected in parallel (see Eq. 8).

$$
I_{\mathrm{inj}}=C_{\mathrm{m}} S \frac{\mathrm{d} V}{\mathrm{~d} t}+I_{\mathrm{KL}}+I_{\mathrm{KH}}+I_{\mathrm{Na}}+I_{\mathrm{H}}+I_{\text {leak }} \text {. }
$$

The currents were driven by the following five ionic conductances: so$\operatorname{dium}\left(g_{\mathrm{Na}}\right)$, low-voltage-gated potassium $\left(g_{\mathrm{KL}}\right)$, high-voltage-gated potassium $\left(g_{\mathrm{KH}}\right)$, hyperpolarization-activated mixed cationic $\left(g_{\mathrm{H}}\right)$, and leak $\left(g_{\text {leak }}\right)$. This model is an extension of the single-compartment VGN model previously described by Hight and Kalluri (2016). Here we varied the conductance density, voltage-activation range, and kinetics of the $I_{\mathrm{H}}$ current to mimic the influence of cAMP, as described by the in vitro characterizations in this study. In addition, we expanded on the previous description of $g_{\mathrm{KL}}$ to include both a Kv1-like and Kv7-like components.

Hyperpolarization-activated cyclic nucleotide-gated mixed-cationic current. Equations 3 through 5 governed the voltage-dependent kinetics of $I_{\mathrm{H}}$ :

$$
\begin{gathered}
I_{H}=\bar{g}_{h} r^{3} S\left(V-E_{h}\right), \\
r_{\infty}=\left[1+\exp \left(\frac{V+V_{1 / 2}}{k}\right)\right]^{-1} \\
\tau_{r}=\frac{\left.\left.\left\{(\exp [-(V+a) / b])^{-1}+\exp [V+a) / d\right]\right]\right\}}{c}+e .
\end{gathered}
$$

Cell surface area $(S)$ was fixed to yield a net capacitance of $10 \mathrm{pF}$. Conductance density $\left(\bar{g}_{h}\right)$, reversal potential $\left(E_{H}\right)$, half-activation voltage $V_{1 / 2}$, slope factor $k$, and voltage-dependent activation kinetics $\left(\tau_{r}\right)$ were based on experimentally derived values from this study. Half-activation voltage and activation kinetics were defined for two conditions; $100 \mu \mathrm{M}$ cAMP and $500 \mu \mathrm{M} \mathrm{dB}$-cAMP, which respectively represent minimum and maximum activation conditions. The parameter values for $V_{1 / 2}, k, a$ and $e$ are summarized in Table 1. Conductance densities and other model parameters are summarized in Tables 2 and 3.

Low-voltage-gated $\mathrm{K}^{+}$currents. Equations 6 through 8 governed the voltage-dependent activation kinetics of $I_{\mathrm{KL}}$; 


$$
I_{K L}=\left(\gamma_{k v 1} \bar{g}_{K L} w_{k v 1}^{4} Z_{k v 1}+\gamma_{k v 7} \bar{g}_{K L} w_{k v 7}^{4} Z_{k v 7}\right) S\left(V-E_{K}\right) .
$$

$I_{\mathrm{KL}}$ was driven in part by Kv1-like and Kv7-like conductances and $\gamma \mathrm{kv} 1$ and $\gamma \mathrm{kv} 7$ defined the fraction of total conductance contributed by each of the two components. The variables $w^{4}$ and $z$ represent activation and inactivation kinetics. The inactivation kinetics were adjusted to reflect the inactivation present in Kv1 channels and the lack thereof in Kv7 channels. The Kv1 component was modeled to activate nearly 10 times faster than the Kv7 component (compare Eqs. 7, 8):

$$
\begin{aligned}
\tau_{w_{k V 1}} & =100\{6 \exp [(V+60) / 6]+16 \exp [-(V+60) / 45]]\}^{-1}+1.5 \\
\tau_{w_{k V 7}} & =1000\{6 \exp [(V+60) / 6]+16 \exp [-(V+60) / 45]]\}^{-1}+1 .
\end{aligned}
$$

Model implementation. The model was implemented in MATLAB $2016 \mathrm{~b}$ running on a Windows 10 operating system. The differential equation (Eq. 2) for the membrane voltage, $V(t)$, was numerically solved using a backward difference method. The associated code is accessible via the ModelDB repository at http://senselab.med.yale.edu/ModelDB/ showModel.cshtml? model $=244202$.

\section{Results}

Whole-cell patch-clamp recordings were made by either rupturedpatch or perforated-patch methods in isolated vestibular ganglion neurons (VGN) from rats ranging in age between postnatal day (P)9 through P21 $(n=146)$. First, we describe the basic firing patterns of VGNs during the second and third postnatal weeks. Second, we define the sensitivity of the $I_{\mathrm{H}}$ current in VGNs to modulation by cyclic adenosine monophosphate (cAMP), a well known modulator of $\mathrm{HCN}$ channels. Third, we describe the influence of $I_{\mathrm{H}}$ on VGN firing patterns and spike-timing regularity. Finally, we explore the interaction between $I_{\mathrm{H}}$ and $I_{\mathrm{KL}}$ using a single compartment conductancebased model for VGN.

\section{Step-evoked firing patterns}

We classified neurons into two broad groups based on the firing patterns evoked by depolarizing current injections: transientfiring ( 83 of 146 neurons; Fig. 1A) and sustained-firing (63 of 146 neurons; Fig. $1 B-D$ ). These classifications are similar to those used by Kalluri et al. (2010) and Iwasaki et al. (2008). Transientfiring neurons displayed strong spike-train accommodation, firing a single spike at the onset of the current step. Beyond spike threshold, the firing pattern of most transient-spiking neurons was invariant to intensity. In a few rare cases, transient-firing neurons fired a second spike when injected with high amplitude currents (4/83 neurons; data not shown). Sustained-firing neurons displayed varying degrees of accommodation, ranging from cells that fired long trains of spikes (sustained-A firing patterns had spikes throughout the depolarizing step; Fig. 1D) to cells with intermediate degrees of accommodation (sustained-B/sustained-C firing patterns had shorter trains of spikes followed by voltage oscillations; Fig. $1 C, B$, respectively). Consistent with Kalluri et al. (2010), the degree of accommodation depended on intensity for the sustainedfiring patterns. Figure $1 E$ shows a sustained-firing pattern at a recording temperature between $35^{\circ} \mathrm{C}$ and $37^{\circ} \mathrm{C}$.

When injected with hyperpolarizing currents, most neurons $(140 / 146)$ responded with a deep voltage hyperpolarization followed by a slow depolarization that eventually reached steady state (Fig. 1A-D). Such hyperpolarization-induced voltage "sags" indicate the presence of the HCN channelmediated current, $I_{\mathrm{H}}$ (Fig. 1C; Rennie and Streeter, 2006; Angelo and Margrie, 2011; Almanza et al., 2012). On average, voltage sags increased in amplitude with larger hyperpolarizing current injections and were first visible when the membrane potential fell below $-80 \mathrm{mV}$.
Our goal was to understand how $I_{\mathrm{H}}$ current influences spiketiming regularity. One way to do this is to compare responses before and after selectively blocking the current with channelspecific antagonists such as ZD7288 and $\mathrm{CsCl}$. This approach was not successful here because both antagonists may affect more than just $\mathrm{HCN}$ channels. For example, $\mathrm{CsCl}$ is known to block other kinds of potassium channels including inward rectifying potassium channels (Bond et al., 1994; Takumi et al., 1995). Previous studies have also shown that ZD7288 can partially block sodium, T-type calcium, and/or potassium channels (Felix et al., 2003; Sánchez-Alonso et al., 2008; Wu et al., 2012). We suspected similar nonspecific effects in VGNs because action potential amplitudes decreased, and net conductance increased when either ZD7288 or CsCl blocked $I_{\mathrm{H}}(5$ of 8 cells; data not shown). This is a counter-intuitive result since blocking the channel should have decreased net conductance. Although not discussed, a similar effect in VGNs is evident in the recordings presented by Yoshimoto et al., 2015. We concluded that we could not rely on these channel antagonists to isolate the role of $I_{\mathrm{H}}$ on firing patterns. As shown next, we chose to amplify $I_{\mathrm{H}}$ by controlling the intracellular concentration of cAMP, which shifts the current's voltage activation range (Almanza et al., 2012).

\section{Enhancing the activation of $I_{H}$ by controlling cAMP concentration}

In voltage-clamp, hyperpolarization produced inward currents with a near instantaneous component $\left(I_{\text {inst }}\right)$ and a slowly activating component that eventually reached steady state $\left(I_{\text {ss }} ;\right.$ Fig. $\left.2 A\right)$. Known $I_{\mathrm{H}}$ antagonists, $\mathrm{CsCl}$ (Fig. 2A2) and ZD7288 (data not shown), blocked the slow current, suggesting that it was $\mathrm{HCN}$ mediated. The current's reversal potential $(-32.7 \pm 2.8 \mathrm{mV}, n=$ 8 ) and voltage activation range and kinetics (discussed further below; Figs. 2, 3) are consistent with reported values for $I_{\mathrm{H}}$ (Biel et al., 2009; Almanza et al., 2012; Yoshimoto et al., 2015). We measured the activation range of $I_{\mathrm{H}}$ under several experimental conditions. First, we varied the concentration of simple cAMP in the intracellular pipette solution (at 10, 100, 200, and $500 \mu \mathrm{M})$. Second, we used perforated-patch methods (where the intracellular milieu is left intact) to estimate the endogenous concentration of cAMP. Third, we used 100 and $500 \mu \mathrm{M}$ db-cAMP, a non-hydrolysable version of cAMP that resists degradation by phosphodiesterases. Fourth, we tested if the activation of $I_{\mathrm{H}}$ is further enhanced by increasing recording temperature, as previously reported in the substantia nigra (Gambardella et al., 2012).

\section{Estimating the voltage-dependent activation of $I_{H}$}

To estimate the voltage-dependent activation properties of $I_{\mathrm{H}}$, we used a voltage-clamp tail protocol, which consists of 1.7-s-long preconditioning steps from -135 to $-35 \mathrm{mV}$ in $5 \mathrm{mV}$ increments followed by a tail step to $-100 \mathrm{mV}$ (Fig. $2 \mathrm{~A}$, bottom). The long preconditioning step allowed $I_{\mathrm{H}}$ to approach steady-state activation but was not so long as to kill cells because of prolonged hyperpolarization (see Materials and Methods). The tail step to $-100 \mathrm{mV}$ was within the typical activation range for HCN channels but hyperpolarized away from the activation range of most other channels known be expressed in VGNs (e.g., low-voltagegated potassium channels). The currents measured during the tail step reflect the channels activated during the preconditioning steps but with a fixed driving force. The maximum current density $\left(I_{\max } /\right.$ capacitance) was larger for sustained-firing neurons than for transient-firing neurons $(11.8 \pm 1.2 \mathrm{pA} / \mathrm{pF}$ vs $6.9 \pm 0.6$ 
A
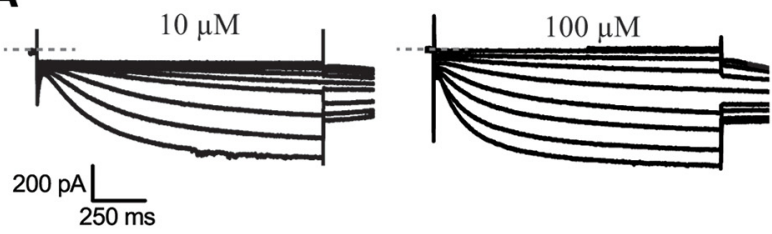

$500 \mu \mathrm{M} \mathrm{db}-\mathrm{cAMP}$

$100 \mu \mathrm{M} 35^{\circ} \mathrm{C}$
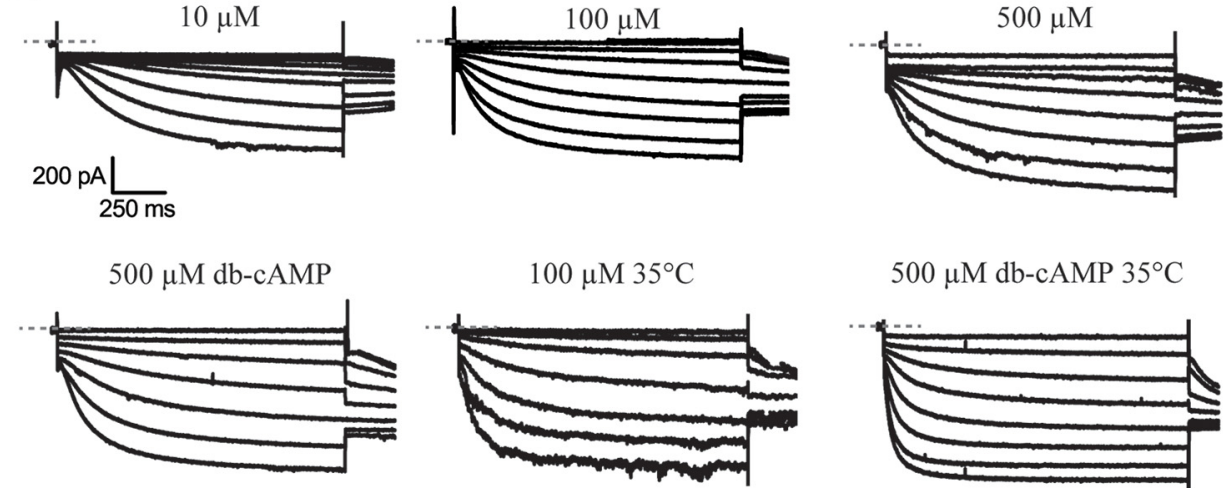

$500 \mu \mathrm{M}$ db-cAMP $35^{\circ} \mathrm{C}$

B

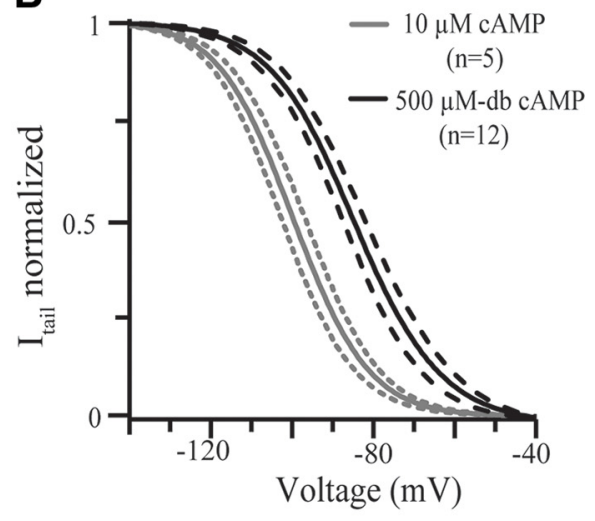

D

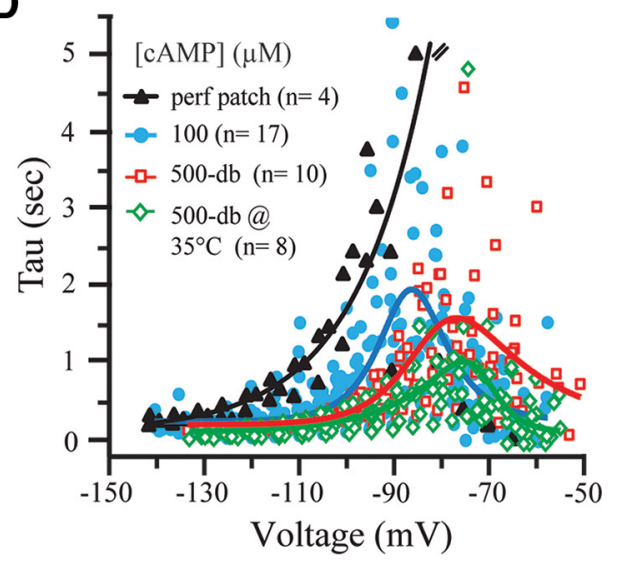

C

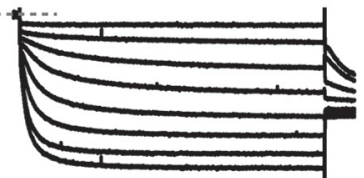

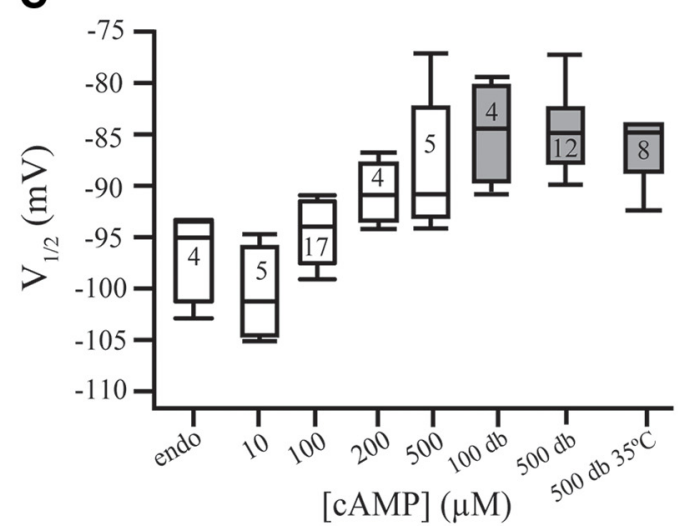

E

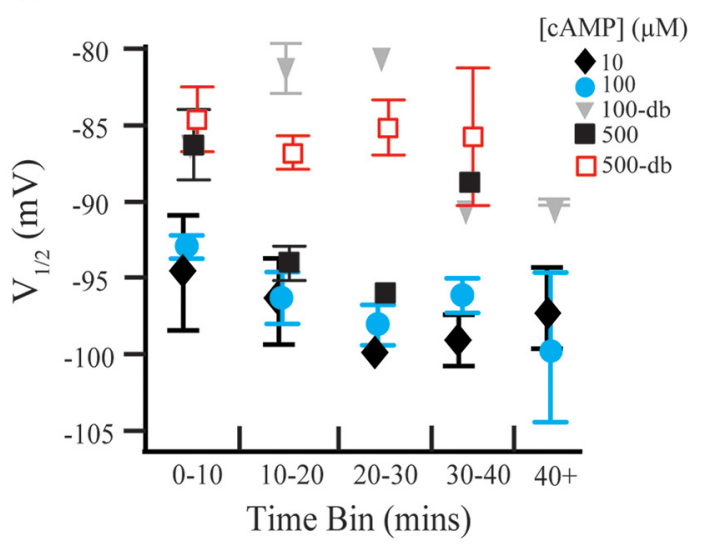

Figure 3. Effects of cAMP concentration and temperature on the activation of $I_{\mathrm{H}}$ currents. $A, I_{\mathrm{H}}$ currents activated by a family of voltage steps from -135 to $-35 \mathrm{mV}$ under several cAMP concentrations and temperature combinations. As in Figure 2, tail currents were used to construct current-voltage relationships. $\boldsymbol{B}$, Normalized current-voltage relationships (means $\pm 95 \%$ confidence intervals) for $I_{H}$ are compared for the $10 \mu \mathrm{M} \mathrm{CAMP}$ and $500 \mu \mathrm{M} \mathrm{db}$-cAMP conditions. The difference between the two conditions illustrates the potential range of $I_{H}$ activation. $C$, Box plots for each cAMP condition. $V_{1 / 2}$ depolarizes dependent on cAMP concentration, db-cAMP conditions produce the most depolarized $V_{1 / 2}$ values (shaded box plots). Increasing the concentration of db-cAMP from 100 to $500 \mu \mathrm{m}$ did not further depolarize $V_{1 / 2}$ and neither did increasing the recording temperature to $35^{\circ} \mathrm{C}$. The endogenous concentration was obtained in perforated patch configuration. Mean slope value $(k)$ for all the conditions was $8.3 \pm 0.2 \mathrm{mV}$ and not affected by cAMP concentration. $D, A$ single exponential was used to fit to the currents produced by voltage steps from -135 to $-65 \mathrm{mV}$. The time constant of the fits, $\tau_{\text {single, }}$ is plotted as function of voltage for $100 \mu \mathrm{m} \mathrm{cAMP}$ (blue; $\left.n=17\right), 500 \mu \mathrm{m} \mathrm{db}$-cAMP (red; $n=10$ ), perforated patch (black; $\left.n=4\right)$, and $500 \mu \mathrm{m} \mathrm{db}$-CAMP at $35^{\circ} \mathrm{C}$ (green; $n=8$ ). Solid lines show fits were produced using Equation 5 to show the relationship of $\tau_{\text {single }}$ values and voltage. Parameters for the fits at $100 \mu \mathrm{m}$ CAMP, $500 \mu \mathrm{M}$ CAMP, and $500 \mu \mathrm{m} \mathrm{db}$-cAMP at $35^{\circ} \mathrm{C}$ are summarized in Table 1. $E$, For all conditions except those with db-cAMP, mean $V_{1 / 2}$ hyperpolarized as a function of time. $V_{1 / 2} \pm S E M$ is plotted as a function of recording time for $10 \mu \mathrm{m}$ cAMP (diamonds), $100 \mu \mathrm{M}$ cAMP (cyan circles), $100 \mu \mathrm{m}$ db-cAMP (gray downward triangle), $500 \mu \mathrm{m}$ cAMP (squares), and $500 \mu \mathrm{m}$ db-cAMP (red open squares).

$\mathrm{pA} / \mathrm{pF} ; t_{(25.8)}=-3.6, p=0.0013$, Welch's unpaired $t$ test), consistent with results reported by Yoshimoto et al. (2015). The normalized tail current $\left(I_{\text {tail }} / I_{\text {tail }}^{\max }\right)$ is plotted as a function of the preconditioning step to show the voltage-dependent activation of $I_{\mathrm{H}}$ (Fig. 2B). The normalized activation curves generated from these whole-cell currents were similar to those extracted by pharmacologically isolating $I_{\mathrm{H}}$ (Fig. $2 B$ ). These results led us to conclude that the tail current measurements were adequate for characterizing $I_{\mathrm{H}}$. All subsequent $I_{\mathrm{H}}$ activation parameters are derived from the unblocked whole-cell currents.

\section{Modulating the voltage-dependent activation range of $I_{H}$}

Examples of whole-cell currents recorded in response to the tail protocol from six cells, each at different intracellular cAMP concentrations and two obtained in $35^{\circ} \mathrm{C}$ are shown in Figure $3 \mathrm{~A}$. The activation curves shifted to more positive potentials as cAMP 
concentration increased; nearly $15 \mathrm{mV}$ when two extreme concentrations were compared ( 10 and $500 \mu \mathrm{M}$ db-cAMP; Fig. 3B). A similar shift has been reported for auditory afferents in response to cAMP analogues (Yi et al., 2010). We found a significant dependence of $\mathrm{V}_{1 / 2}$ on cAMP condition $\left(F_{(6,44)}=11.67, p=0.0001\right.$ based on one-way ANOVA; Fig. $3 C$ ). Based on similarity in $V_{1 / 2}$, we estimate that the endogenous concentration of cAMP (the perforated-patch condition) is between 10 and $100 \mu \mathrm{M}$. This estimate is consistent with reports by Almanza et al. (2012). At 100 $\mu \mathrm{M}$ cAMP, the mean $\mathrm{V}_{1 / 2}$ and slope factor $(k)$ are $-94.32 \pm 3.06$ $\mathrm{mV}$ and $7.97 \pm 1.31 \mathrm{mV}(n=14)$, respectively. This is also consistent with reported values for $I_{\mathrm{H}}$ at $100 \mu \mathrm{M}$ cAMP $(-93 \pm 2$ and $9 \pm 0.9 \mathrm{mV}$; Almanza et al., 2012). Interestingly, mean $\mathrm{V}_{1 / 2}$ values were not significantly different between the 100 and 500 $\mu \mathrm{M} \mathrm{db}$-cAMP conditions, suggesting that enzymatic degradation of cAMP is a critical parameter affecting cAMP concentration and $I_{\mathrm{H}}$ activation.

Temperature did not further depolarize the activation range of $I_{\mathrm{H}}$. In the substantia nigra increasing recording temperature depolarizes the activation range of $I_{\mathrm{H}}$ by as much as $15-20 \mathrm{mV}$ (Gambardella et al., 2012). Here we found that recording in 500 $\mu \mathrm{M} \mathrm{db}$-cAMP with an elevated temperature $\left(35^{\circ} \mathrm{C}\right)$ did not further depolarize the activation range of $I_{\mathrm{H}}$ (Fig. $3 C$, compare gray box plots). These results suggest that the db-cAMP is at least as effective at shifting the activation range of $I_{\mathrm{H}}$ as temperature. We did not test the influence of temperature alone on the activation range of $I_{\mathrm{H}}$, although such a temperature-dependent shift would offer a physiologically plausible mechanism for enhancing $I_{\mathrm{H}}$.

\section{Modulating the activation time course}

Next, we show that the time course of $I_{\mathrm{H}}$ activation speeds up with increased concentrations of cAMP. We applied single exponential fits to the currents evoked by the family of voltage steps from -135 to $-55 \mathrm{mV}$. A single exponential reliably fit our data between -135 and $-55 \mathrm{mV}$. Double exponentials better fit the $I_{\mathrm{H}}$ current at $-135 \mathrm{mV}$ (Almanza et al., 2012; Liu et al., 2014) but failed for the smaller hyperpolarizations as the size of $I_{\mathrm{H}}$ current diminished. We relied on single exponential fits because they were robust in all cells and for wide range of voltage steps. In Figure $3 D$, the exponent $\tau$ is plotted as a function of voltage steps for recordings made under the four cAMP conditions (perforated-patch, black triangle; $100 \mu \mathrm{M}$ cAMP, blue circle; 500 $\mu \mathrm{M}-\mathrm{dB}$ cAMP, red square; $500 \mu \mathrm{M}-\mathrm{dB}$ cAMP @ $35^{\circ} \mathrm{C}$, green triangles). The voltage-dependent trends for $\tau$ activation were fit with Equation 5 (see Materials and Methods) for each cAMP condition (Fig. 3D, solid lines). These same fits were used in the model described later. In each condition, the current activates fastest at extreme hyperpolarizations and slows as the voltage step approaches $\mathrm{V}_{1 / 2}$. As the voltage steps become more positive the activation kinetics speed up. Like the cAMP-dependent shift in voltage activation, the activation kinetics shift such that at any particular voltage (e.g., $-90 \mathrm{mV}$ ) the $500 \mu \mathrm{M} \mathrm{db}$-cAMP was the fastest. Similar cAMP driven acceleration in activation kinetics have been reported in various types of neurons including in chick nucleus laminaris, substantia nigra pars compacta and spiral ganglion (Yamada et al., 2005; Yi et al., 2010; Gambardella et al., 2012). Although increasing temperature did not further depolarize half activation voltage beyond that produced by $500 \mu \mathrm{M} \mathrm{dB}-$ cAMP (Fig. 3C), it did speed up activation kinetics (Fig. 3D, green symbols).

\section{Time-dependent stability of $I_{H}$ modulation by cAMP}

Although we found that the activation range of $I_{\mathrm{H}}$ could be shifted by increasing cAMP concentration, the shifts were most robust and prolonged in the $\mathrm{AB}$-cAMP conditions. Since we were characterizing the activation properties of $I_{\mathrm{H}}$ and firing patterns in the same groups of cells, it was important for us to maintain stability for $\sim 30-40 \mathrm{~min}$. To determine the time-stability of cAMP driven shift, we measured $I_{\mathrm{H}}$ activation curves at regular time intervals throughout the recording session. Figure $3 E$ shows mean $\mathrm{V}_{1 / 2}$ as a function of recording time and by cAMP condition. For all but the $500 \mu \mathrm{M} \mathrm{dB}$-cAMP condition, the mean $\mathrm{V}_{1 / 2}$ was most depolarized during early recordings $(0-10 \mathrm{~min})$ and became progressively more hyperpolarized as neurons were held for longer periods (30-40 min). In Figure 3C, activation parameters of $I_{\mathrm{H}}$ were taken from the earliest recording time possible. In $\mathrm{db}$-cAMP, $\mathrm{V}_{1 / 2}$ values remained time stable, showing no significant difference between early $(0-10 \mathrm{~min})$ and late recordings $\left(30-40 \mathrm{~min} ; t_{(3.3)}=-0.13, p=0.907\right.$, paired $t$ test $)$. This is presumably because $\mathrm{db}$-cAMP is a non-hydrolysable version of cAMP that can maintain elevated intracellular cAMP levels for hours (Campos-Toimil et al., 2008). We did not determine whether $\mathrm{db}$-cAMP remains stable beyond $40 \mathrm{~min}$. The ability to stably shift the activation range of $I_{\mathrm{H}}$ was ultimately important for characterizing the influence of $I_{\mathrm{H}}$ on firing patterns (presented in the next section). Most of our quantitative comparisons are between the $100 \mu \mathrm{M}$ cAMP condition (which we estimate to be most like the endogenous condition) and the $500 \mu \mathrm{M} \mathrm{db}$-cAMP condition (where we are confident that $I_{\mathrm{H}}$ activation was the most robust and stable).

\section{Effects of cAMP on firing patterns}

Step-evoked firing patterns with increased cAMP

To understand how cAMP driven enhancement of $I_{\mathrm{H}}$ influenced firing patterns, we compared spikes evoked by $\sim 100-120$ pA step currents in $100 \mu \mathrm{M}$ cAMP $(n=12)$ and $500 \mu \mathrm{M} \mathrm{db}-\mathrm{cAMP}(n=$ $10)$. We also recorded at $35^{\circ} \mathrm{C}$ with $500 \mu \mathrm{M} \mathrm{cAMP}(n=8)$ to test whether increasing temperature together with elevated cAMP concentrations had an additional influence on spike timing. Figure 4, $A 1$ and $A 2$, shows spike traces from example sustainedspiking neurons in the three recording conditions. For each neuron, we quantified the first spike latency $\left(t_{\mathrm{p}}\right)$ and the slope of the afterhyperpolarization trajectory (AHP slope; Fig. 4B1,B2, filled symbols are for the examples in $A$ ). We applied two-way ANOVAs to test the influence of the three cAMP conditions and firing-pattern category on each of the two spike features. Both, first spike latency and AHP slope were significantly dependent on cAMP condition $\left(F_{(2,29)}=4.47, p=0.02 ; F_{(2,30)}=5.10, p=0.01\right.$, respectively) and AHP slope also depended on firing-pattern $\left(F_{(1,30)}=7.58, p=0.01\right)$. Post hoc analysis by Tukey HSD indicated that first spike latency tended to be shorter and AHP slopes were steeper in the two $500 \mu \mathrm{M} \mathrm{db}$-cAMP conditions than in the $100 \mu \mathrm{M}$ cAMP condition $(p=0.030$ and $p=0.086$, respectively, for first spike latency; $p=0.026$ and $p=0.031$, respectively, for AHP slope). Neither feature was significantly different between the $500 \mu \mathrm{M} \mathrm{db}$-cAMP and $500 \mu \mathrm{M} \mathrm{db}$-cAMP at $35^{\circ} \mathrm{C}$ conditions ( $p=0.96$ for first spike latency; $p=0.99$ for AHP slope). These results suggest that more $I_{\mathrm{H}}$ current was available in elevated cAMP to speed up afterhyperpolarization trajectories and spike timing, but that raising temperature had no additional influence on these two spike features (Fig. $4 B, D$ ).

Noise ( pseudo-EPSC) driven firing patterns with increased cAMP Next, we examined whether the enhanced activation of $I_{\mathrm{H}}$ yielded regular spiking. Although spontaneous spiking in vivo is driven 
A1

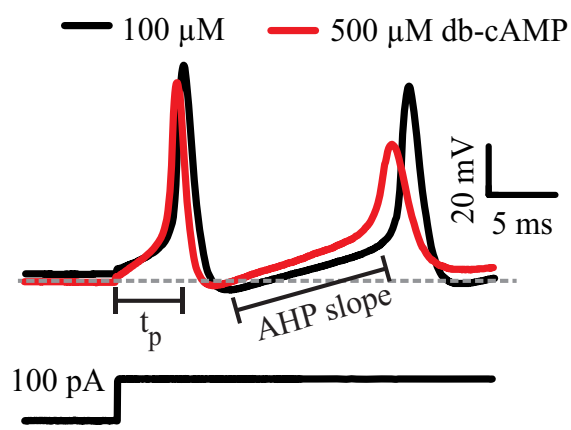

A2

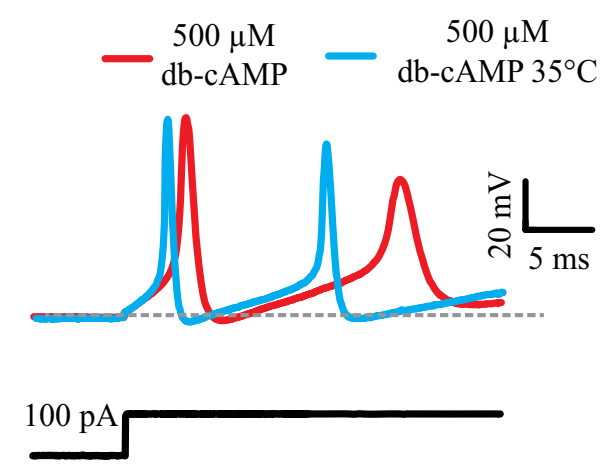

B1

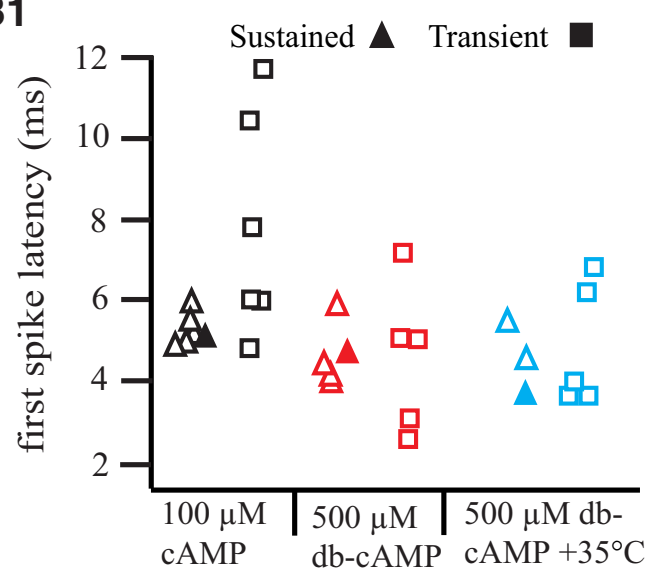

B2

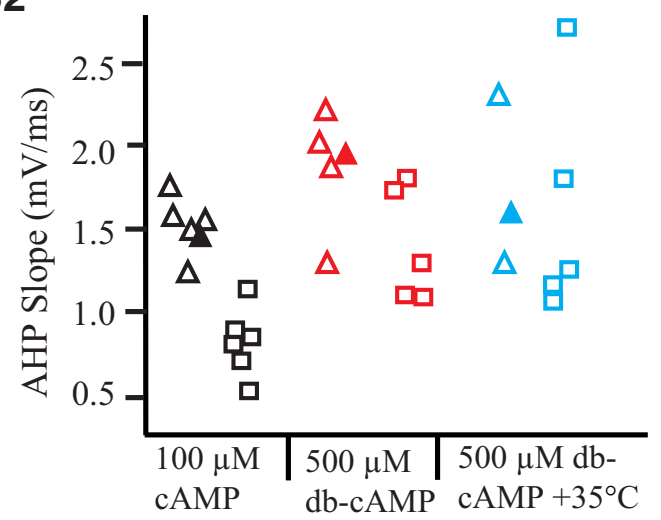

Figure 4. The effects of a db-CAMP and temperature on step-evoked firing patterns. A1, Example responses to a depolarizing current step for sustained-firing VGNs; one recording in $100 \mu \mathrm{Mm}$ CAMP (black) and the second in $500 \mu \mathrm{m} \mathrm{db}-\mathrm{cAMP}$ at $25^{\circ} \mathrm{C}$ (red) conditions. A2, Example responses to a depolarizing current step for sustained-firing VGNs; one recording in $500 \mu \mathrm{m} \mathrm{db}$-cAMP at $25^{\circ} \mathrm{C}$ (red) condition and the second in $500 \mu \mathrm{m} \mathrm{db}$-CAMP at $35^{\circ} \mathrm{C}(\mathrm{cyan})$ condition. VGN firing patterns in A were obtained in response to similar current step $(\sim 100-120 \mathrm{pA})$. First spike latency $\left(t_{\mathrm{p}}\right)$ and the slope of the afterhyperpolarization (AHP) trajectory were obtained as shown in A1. AHP slope was obtained by taking the slope of a line fit to the region illustrated in $A$. Dashed gray line shows $-60 \mathrm{mV}$. $B 1, B 2$, Data for time to peak (ms) and AHP slope ( $\mathrm{mV} / \mathrm{ms}$ ) for transient $(\square)$ and sustained $(\Delta)$ is shown for all three conditions $100 \mu \mathrm{m} \mathrm{CAMP} \mathrm{(black} n=11)$ and $500 \mu \mathrm{m} \mathrm{db}-\mathrm{cAMP}$ at both $25^{\circ} \mathrm{C}(\mathrm{red}$; $n=10)$ and $35^{\circ} \mathrm{C}($ cyan; $n=8)$. Solid symbols are data for the example VGN shown in $A$.

by quantal release of neurotransmitter from hair-cells, dissociated VGNs require current injection to induce spiking. To mimic the random timing of synaptic input, we injected "pseudosynaptic" currents into VGN somata ( $n=47$; for additional detail see Materials and Methods; Kalluri et al., 2010). The size of the unitary EPSC was varied to drive neurons at different rates (Fig. 5A, $B$ ). Moreover, the average EPSC size needed to drive neurons to similar rates was larger in transient-firing neurons than sustained-firing neurons (Fig. 5C). Based on the steeper AHPs in step-evoked firing patterns, we hypothesized that firing rates should be higher in $500 \mu \mathrm{M} \mathrm{db}$-cAMP where the enhanced activation of $I_{\mathrm{H}}$ contributed to the depolarization phase of spike generation. Recordings with $500 \mu \mathrm{M}$ db-cAMP $(n=11)$ produced a mean firing rate of $30.4 \pm 2.9$ spikes/s, significantly higher than the $18.5 \pm 1.0$ spikes/s in the $10 / 100 \mu \mathrm{M}$ condition $(p<0.001$, Tukey HSD). However, when we looked at each condition by firing type we saw that this increase in mean firing rate was mainly driven by sustained-B-firing neurons $(n=5)$, which produced firing rates as high as 70 spikes/s in db-cAMP (Fig. 5C, black triangles). Again, increasing the temperature from $25^{\circ} \mathrm{C}$ to $35^{\circ} \mathrm{C}$ did not further increase mean firing rates beyond what was achieved with db-cAMP alone $(32.6 \pm 3.2$ spikes $/ \mathrm{s}, n=$ 8 vs $30.4 \pm 2.9$ spikes/s, $n=11$ ).

Next, we quantified the regularity of spike timing with the CV of the ISI histograms (Fig. 5B). Smaller CV values correspond to narrower histograms and more regularly-timed spike intervals. Figure $5 D$ plots $C V$ as a function of mean ISI measured for all cAMP conditions for $(n=47)$, of which eight were in $500 \mu \mathrm{M}$ db-cAMP between $35^{\circ} \mathrm{C}$ and $37^{\circ} \mathrm{C}$. Recall (from Materials and Methods) that we drove each cell to spike at different rates to allow us to compare CV across neurons at similar spike rates. Consequently, there are $>47$ points on the plot because each cell contributes more than one point.

We conducted a slope regression analysis to test the influence of ISI and CAMP condition on CV. Spike-train regularity was significantly dependent on ISI $\left(F_{(1,130)}=7.015, p=\right.$ $0.0092)$ but not on cAMP condition $\left(F_{(4,130)}=0.32, p=\right.$ $0.8624)$. In other words, this analysis suggests that once we account for the effect that increasing cAMP has on ISI (recall from Fig. $5 C$ that cAMP condition does affect spike rate or the inverse of ISI), cAMP has no further influence on regularity. For example, even in $500 \mu \mathrm{M} \mathrm{db}$-cAMP, were the activation range of $I_{\mathrm{H}}$ is most depolarized and ISIs were as short as $10 \mathrm{~ms}$, firing patterns were still very irregular (with CV values $>0.45$; Fig. $5 D$, blue triangles). This is counter to the findings in neonatal calyx terminals (Horwitz et al., 2014), where increasing cAMP or blocking $I_{\mathrm{H}}$ reduced $\mathrm{CV}$ by an order of magnitude (ranging from $\mathrm{CV}=0.8-0.08$ ).

Increasing the temperature from $25^{\circ} \mathrm{C}$ to $35^{\circ} \mathrm{C}$ in $500 \mu \mathrm{M} \mathrm{db}$ cAMP did not significantly change mean firing rates $(30.4 \pm 2.9$ 
A

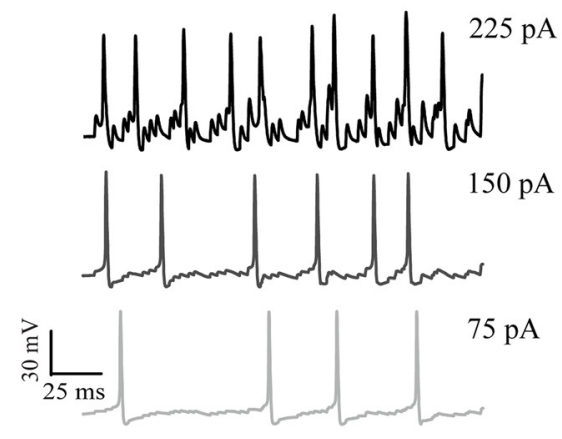

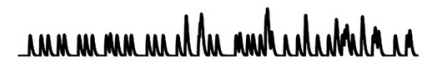

C

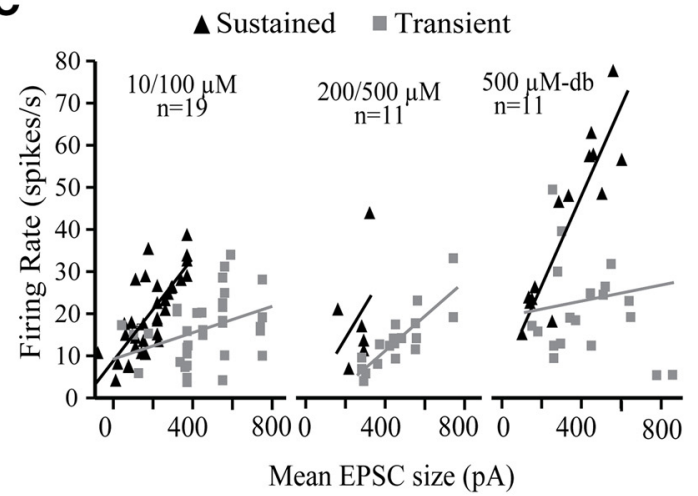

B

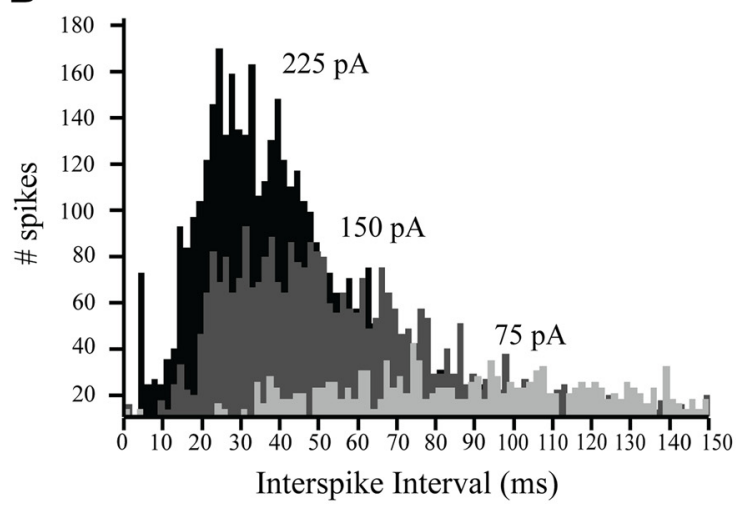

D

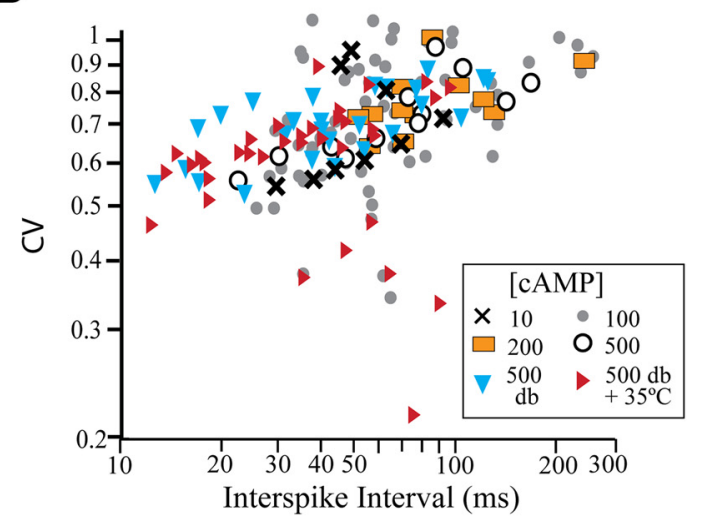

Figure 5. Enhancing $I_{H}$ activation by increasing cAMP concentration does not increase spike-timing regularity. $A$, Spike trains in response to pseudo-synaptic current injection. Spike rate increased as the amplitude of unitary pseudo EPSC increased. $\boldsymbol{B}$, ISI histograms constructed from spike trains in response to frozen variations of pseudo-synaptic currents. Mean spike interval (ISI) decreased as unitary EPSC amplitude increased compare black and gray histograms. C, Firing rate (spikes/s) as a function of mean EPSC amplitude (pA) in different cAMP concentrations. Mean firing rate was impacted by cAMP condition and mean EPSC amplitude. $D, C V$ as a function of mean ISI (ms). Symbols represent cAMP conditions: $10 \mu \mathrm{M}$ CAMP ( $n=4 ; \mathrm{X}), 100 \mu \mathrm{M}$ CAMP ( $n=12 ; \mathrm{gray})$, $200 \mu \mathrm{M}$ cAMP ( $n=5$; orange rectangle), $500 \mu \mathrm{m} \mathrm{cAMP}(n=6$; black $\bigcirc), 500 \mu \mathrm{m} \mathrm{db}$-cAMP $(n=12 ;$ blue $\nabla)$, and $500 \mu \mathrm{m} \mathrm{db}$-cAMP at $35^{\circ} \mathrm{C}(n=8 ;$ red $>$ ). Fourty-seven cells contributed to this plot, and an individual cell could have contributed multiple points.

spikes/s, $n=11$ vs $32.6 \pm 3.2$ spikes/s, $n=8 ; p=0.56)$. There is significant overlap between the red triangles and blue triangles in Figure $5 D$, except for a few spike trains with $C V<0.5$ (which we discuss in the next section). These results suggest that regular spike trains do not emerge even with faster channel kinetics at higher temperature.

Overall, firing patterns were more irregular here than observed by Kalluri et al. (2010), who recorded from neonatal VGNs without explicitly controlling the activation of $I_{\mathrm{H}}$. Thus, although activation of $I_{\mathrm{H}}$ via cAMP was sufficient to increase firing rates, it did not produce highly regular firing.

\section{Developmental changes in firing patterns indicate acquisition of $I_{\mathrm{KL}}$}

An explanation for why VGNs failed to produce highly regular firing can be seen by examining the firing pattern of each cell in response to step currents. In Figure $6 \mathrm{~A}$, we recolored the symbols in Figure $5 D$ according to step-evoked firing pattern. Triangles indicate recordings made between $35^{\circ} \mathrm{C}$ and $37^{\circ} \mathrm{C}$. Transientfiring neurons (Fig. 6A) had the highest $\mathrm{CV}$ values (blue symbols), sustained-B firing neurons had intermediate $\mathrm{CV}$ values (gray symbols), and sustained-A firing neurons had the lowest $\mathrm{CV}$ values (red symbols). One sustained-A spiking neuron encountered in $500 \mu \mathrm{M} \mathrm{db}$-cAMP at $35^{\circ} \mathrm{C}$ produced the most regular spike train in this study and was similar to that seen for sustained-A neurons by Kalluri et al. (2010) (Fig. 6A, red triangle with $\mathrm{CV} \sim 0.2$ ). This suggests that the firing pattern is the dominant predictor for spike-train regularity.

The above described firing-pattern-dependent stratification in $\mathrm{CV}$ is qualitatively consistent with that previously reported in younger VGNs (Kalluri et al., 2010) but is quantitatively very different. The overall range of CV values is much more compressed within the irregular/high CV region at the older ages in this study than at younger ages (shaded regions indicate the range of CVs reported by Kalluri et al., 2010). One striking difference between the two studies is that here we saw fewer cells that responded to current injections with sustained-A spike patterns, which appears to be a developmentally driven change. Figure $6 B$ illustrates that the proportion of VGNs that fire with sustainedfiring patterns diminishes during the first 3 postnatal weeks. During the first postnatal week, equal numbers of cells had either transient-firing or sustained-firing patterns (Fig. 6B); among the sustained-firing group $\sim 12 \%$ had a fully sustained-A firing pattern. These proportions are very different by the third postnatal week when nearly $70 \%$ of cells had transient-firing patterns with very few cells having sustained-A firing patterns $(\sim 5 \%)$.

A shift toward the more phasic sustained-B-spiking and transient-spiking patterns is consistent with an increase in the expression of low-voltage-gated potassium currents (Iwasaki et al., 2008; Kalluri et al., 2010; Yoshimoto et al., 2015). Also, consistent with this suggestion, we found that there was an agedependent hyperpolarization in resting potential $\left(V_{\text {rest }}\right)$ and 


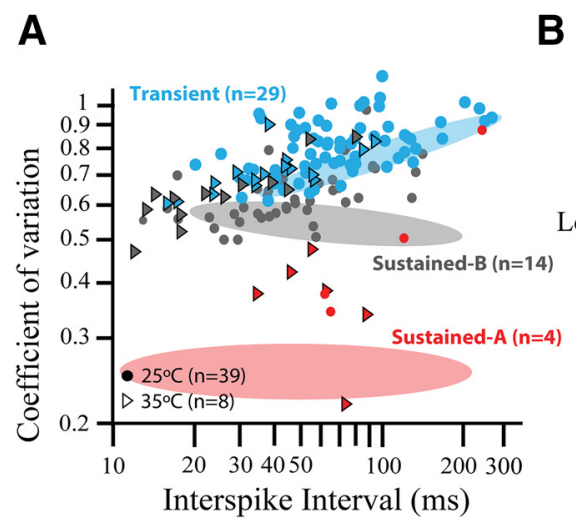

C

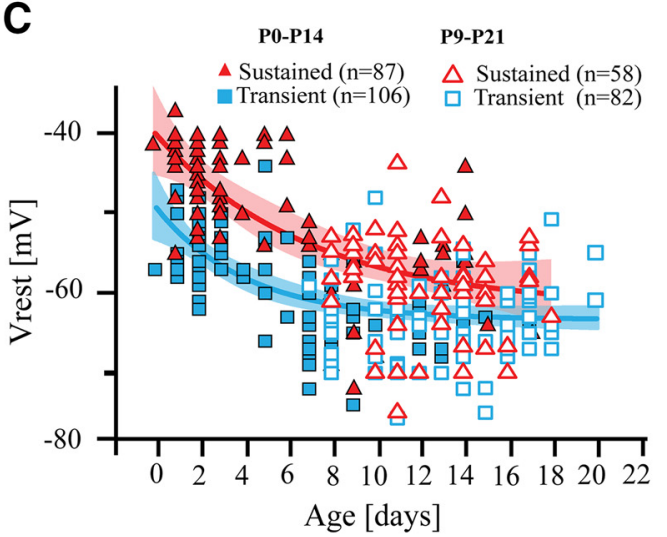

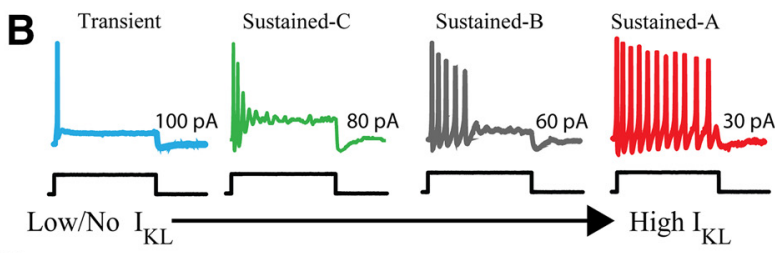
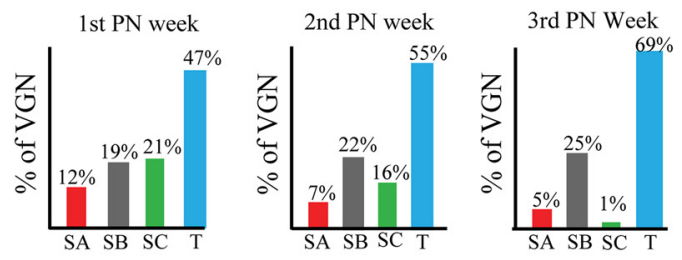

D

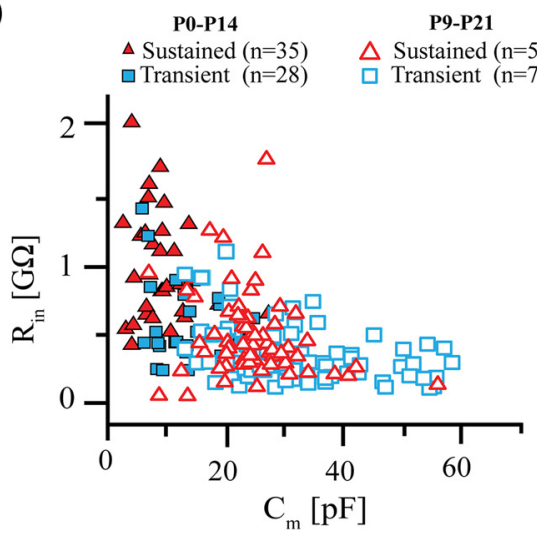

Figure 6. Age-dependent changes in firing pattern and passive membrane properties. $A$, The influence of firing-type on spike-timing regularity. Same data as in Figure $5 D$ but separated by step-evoked firing pattern; transient-firing (blue; $n=29$ ), sustained-B (gray; $n=14$ ), and sustained-A (red; $n=4)$. Data obtained in $35^{\circ} \mathrm{C}$ are distinguished by the symbol $(n=8)$. Shaded regions represent the $C V$ range reported by Kalluri et al. (2010) before the first 2 postnatal weeks. $\boldsymbol{B}-\boldsymbol{D}$, Data from the present study from more mature neurons (open symbols; $n=130$ ) are pooled with that from the younger cells reported by Kalluri et al. (2010) (solid symbols; $n=192$ ). $\boldsymbol{B}$, Examples of $V G N$ firing types in response to a 750 ms long current step. Bar plots showing the proportion of cells that fall into each firing-pattern group from the first, second, and third postnatal weeks. C, Resting potential becomes more negative with age for both firing types. During early postnatal days, the resting potential for sustained-firing cells (red triangles) were more depolarized than for transient-firing cells (blue squares). As indicated by the convergence of the two trend lines, this difference diminished at older ages. Trend lines are exponential fits to the pooled dataset with $95 \%$ confidence intervals (shaded regions) for transient (blue) and sustained (red) firing neurons $\boldsymbol{D}$, Input resistance $\left(R_{\mathrm{in}}\right)$ as a function of membrane capacitance $\left(C_{\mathrm{m}}\right)$, for 56 sustained and 71 transient neurons. On average, $C_{\mathrm{m}}$ is larger for older neurons, regardless of firing pattern. $R_{\text {in }}$ becomes smaller for older sustained-spiking neurons. Together, $\boldsymbol{A}-\boldsymbol{D}$ follow the idea that sustained-spiking cells are acquiring additional potassium currents as they mature.

decrease in input resistance $\left(R_{\text {in }}\right)$ for all cell types. In Figure 6, $C$ and $D$, we pooled data from the present study $(n=146)$ with those from Kalluri et al. (2010) $(n=192)$ to illustrate the agedependent changes in these passive membrane properties. Resting potentials of sustained-spiking (red) and transient-spiking (blue) VGNs were different during early postnatal days but begin to converge with that of transient firing neurons by the third postnatal week (Fig. $6 C$ ). Membrane capacitance $\left(C_{\mathrm{m}}\right)$ was typically larger in the older dataset (Fig. $6 D$ ) with transient-firing neurons being larger $(40.9 \pm 1.4 \mathrm{pF}, n=77)$ than sustainedfiring neurons $\left(31.4 \pm 1.7 \mathrm{pF}, n=60 ; t_{(132.3)}=4.45 p=0.0001\right.$, unpaired $t$ test). When compared by cell size, $R_{\text {in }}$ values were smaller in transient-firing neurons than sustained-firing neurons at younger ages (Fig. 6D, red triangles, blue squares,), but the differences are less striking at older ages. These data suggest that the ion channel properties of VGNs are becoming less heterogeneous with development. This developmental shift in firing patterns likely accounts for why we did not see highly regular firing, despite enhancing the activation of $I_{\mathrm{H}}$.

\section{Modeling the influence of $I_{\mathrm{H}}$ activation in the presence and absence of $I_{\mathrm{KL}}$}

Our initial prediction was that by recording from older ages and increasing the activation of $I_{\mathrm{H}}$ we would have seen increased
sustained-A spiking in response to current steps and more regular spiking in response to pseudo-synaptic stimulation. However, on average, we saw the opposite result in that VGN responses shifted toward transient/irregular spiking. Next, we will show through model simulations that this result can be accounted for by considering the interaction between $I_{\mathrm{KL}}$ and $I_{\mathrm{H}}$, both of which are present in nearly all VGNs at older ages.

We used a single-compartment conductance-based vestibular ganglion model in which we could control the conductance density of $I_{\mathrm{KL}}$ and the activation of $I_{\mathrm{H}}$ (see Materials and Methods; Hight and Kalluri, 2016). Sustained-A and sustained-B firing patterns were simulated (Fig. 7A1,B1) by setting $g_{\mathrm{KL}}$ to 0 and 0.65 $\left(\mathrm{mS} / \mathrm{cm}^{2}\right)$, respectively. The equations describing $g_{\mathrm{KL}}$ are the same as those described by Hight and Kalluri (2016) for currents carried through an equal combination of Kv1 and Kv7 type ion channels. Sodium channel density $\left(g_{\mathrm{Na}}\right)$ was set to $20 \mathrm{mS} / \mathrm{cm}^{2}$ for all simulations. This value was large enough to avoid spike train adaptation because of sodium channel inactivation (Hight and Kalluri, 2016). A family of $1500 \mathrm{~ms}$ step depolarizing currents was used to elicit spike trains for each condition. Figure 7, $A 1$ and $B 1$, shows responses at current threshold. The conductance density for the HCN channel, $g_{\mathrm{H}}$, was set from 0.07 through $0.91 \mathrm{mS} /$ $\mathrm{cm}^{2}$. This range is between 0.5 and 7 times the mean value we measured in VGNs $\left(0.13 \mathrm{mS} / \mathrm{cm}^{2}\right)$. Here we show the result for 
A1

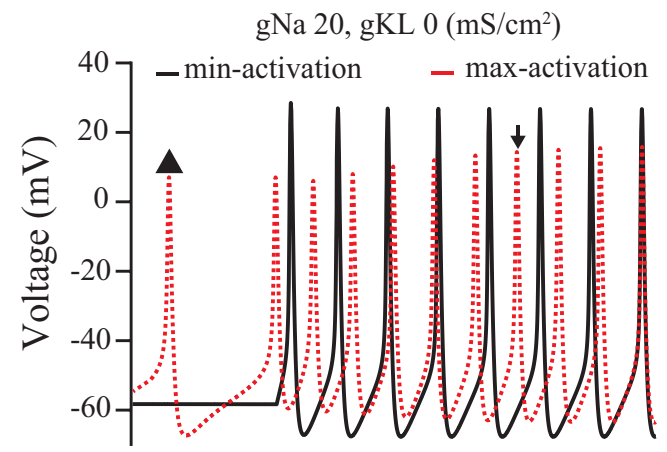

A2

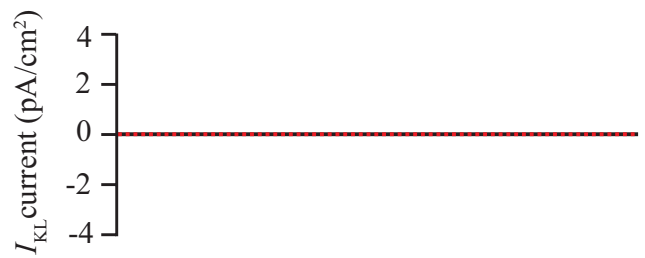

A3

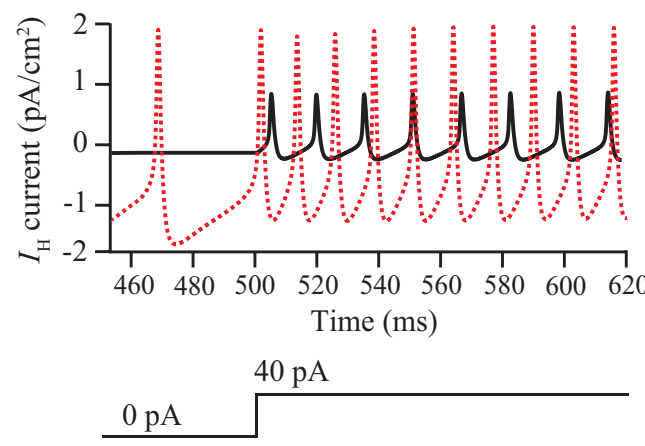

B1

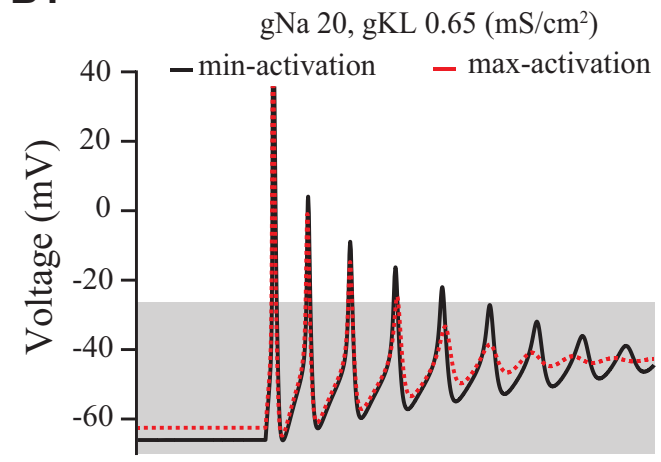

B2

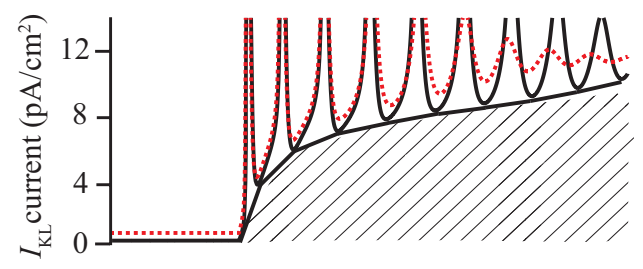

B3

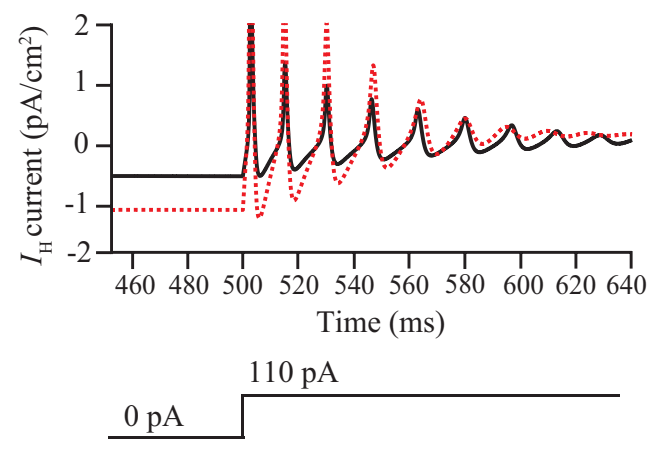

Figure 7. The influence of shifting the activation range of $I_{H}$ explored in a conductance-based model. Model results from sustained-A and $-B$ type VGNs in response to a $1500 \mathrm{~ms}$ depolarizing current step $\left(\boldsymbol{A} 3, \boldsymbol{B} 3\right.$, bottom). Two $I_{H}$ conditions are shown in each panel; minimum activation (black) and maximum activation (dashed red). $\boldsymbol{A}, \boldsymbol{B}$, The sustained-A type model was produced by setting $g_{\mathrm{N}}$ a to $20 \mathrm{mS} / \mathrm{cm}^{2}$ and $g_{\mathrm{KL}}$ to 0 . The sustained-B type model was produced by setting $g_{\mathrm{Na}}$ to $20 \mathrm{mS} / \mathrm{cm}^{2}$ and $g_{\mathrm{KL}}$ to $0.65 \mathrm{mS} / \mathrm{cm}^{2}$ composed equally of Kv1 and Kv7. A1, B1, Voltage-clamp responses for the sustained-A and $-B$ models under model conditions for $I_{H}$ current (minimum activation, where $V_{1 / 2}=-96 \mathrm{mV}$ and maximum activation, where $V_{1 / 2}=-84 \mathrm{mV}$. Black triangle $(\mathbf{\Delta})$, The max activation produced spontaneous firing at rest. $B$, Sustained-B neurons exhibit accommodation as voltage oscillations are present in all $/{ }_{H}$ conditions after $\sim 60 \mathrm{~ms}$ following the onset of the stimulus. $\boldsymbol{A 2}, \boldsymbol{B} 2, I_{\mathrm{KL}}$ current $\left(\mathrm{pA} / \mathrm{cm}^{2}\right)$ as a function of time for $\boldsymbol{A}$ and the shaded region in $\boldsymbol{B}$. $\boldsymbol{A} 2$, No $I_{\mathrm{KL}}$ current is seen because $g_{\mathrm{KL}}$ is set to 0 for the sustained-A type VGN. $\boldsymbol{B}$, The $I_{\mathrm{KL}}$ current at rest and during the AHP grows larger as $I_{\mathrm{H}}$ is activated. Hatched region, To estimate the net charge $\left(\mathrm{Q} / \mathrm{cm}^{2}\right)$ carried by $I_{\mathrm{KL}}$ we took the sum of the area under each minimum, which is proportional to the shaded region. $\boldsymbol{A} \mathbf{3}, \boldsymbol{B}, I_{\mathrm{H}}$ current $\left(\mathrm{pA} / \mathrm{cm}^{2}\right)$ as a function of time for $\boldsymbol{A}$ and the shaded region in $\boldsymbol{B}$. $\boldsymbol{A} \mathbf{B}-\boldsymbol{B} \mathbf{3}$, As expected the $I_{\mathrm{H}}$ current is larger at rest and throughout the stimulus for max activation cases than for the minimum-activation case. Note that because of the slow kinetics of the HCN channel, the current of $I_{H}$ switches from inward $(-)$ to outward $(+)$ during the upswing of the spike.

the largest value because conductance density for $I_{\mathrm{H}}$ is reported to be larger in calyx terminals than in somata (Horwitz et al., 2014). We repeated simulations with two $I_{\mathrm{H}}$ conditions that represented the current's most hyperpolarized and depolarized voltage-dependent activation (minimum and maximum activation, representative of the $100 \mu \mathrm{M}$ cAMP and $500 \mu \mathrm{M}$ db-cAMP conditions, respectively). The parameters describing the voltage activation range and kinetics for these conditions were based on the data shown in Figure 3 and are represented by Equations 3-5 (see Materials and Methods; parameters are summarized in Table 1).

Activation of $I_{\mathrm{H}}$ excites faster spiking in the absence of $I_{\mathrm{KL}}$ Figure $7 A 1-A 3$ shows the firing patterns and flow of currents through $g_{\mathrm{H}}$ and $g_{\mathrm{KL}}$ for the sustained-A simulation. Only the beginning of each spike train is shown to emphasize the details of spike shape and timing. Figure 7, $A 2$ and $A 3$, shows the flow of $I_{\mathrm{H}}$ and $I_{\mathrm{KL}}$ currents into and out of the cell (negative and positive currents, respectively). When $I_{\mathrm{H}}$ was minimally activated, very little $I_{\mathrm{H}}$ current flows into the cell before current injection begins (i.e., the resting phase; Fig. 7A3). During current injection, the membrane potential depolarizes and the model produces spikes throughout the current step. Between each spike (as the cell emerges from the afterhyperpolarization), a little $I_{\mathrm{H}}$ current flows into the cell when the membrane potential is more negative than the reversal potential for $I_{\mathrm{H}}(-40 \mathrm{mV})$. When the membrane potential crosses the reversal potential, $I_{\mathrm{H}}$ current flows out of the cell. When the activation range of $I_{\mathrm{H}}$ is shifted in the maximum activation condition (dashed trace), there is significant $I_{\mathrm{H}}$ flowing into the cell during rest and between spikes. As a result of this extra depolarizing current, the model begins to spike spontaneously (Fig. 7A1, black triangles). The flow of inward and outward currents through the $I_{\mathrm{H}}$ conductance is also larger during the current injection resulting in shorter mean intervals between spikes ( $11.97 \pm 0.12 \mathrm{vs} 12.32 \pm 0.01 \mathrm{~ms})$ and more evoked spikes 
during the step (139 vs 122 spikes). Also note that the spikes are much shorter in the maximum activation condition because the slow inactivation of $I_{\mathrm{H}}$ means that the channel remains open during the action potential, adding another large repolarizing potassium current past its reversal potential. Note that because of the spontaneous-spiking, the increase in spike rate during the current step is not immediately visible but, becomes more obvious by $\sim 60 \mathrm{~ms}$ after stimulus onset (black arrow). Overall, the excitatory influence of $I_{\mathrm{H}}$ is consistent with the pace-making role of this current in cardiac cells (DiFrancesco, 2010) and with previous suggestions for vestibular afferents (Horwitz et al., 2014; Yoshimoto et al., 2015).

\section{Activation of $I_{\mathrm{H}}$ dampens spiking in the presence of $I_{\mathrm{KL}}$}

The influence of $I_{\mathrm{H}}$ on firing patterns is different in the presence of $I_{\mathrm{KL}}$. In Figure $7 B 1-B 3$, we show a simulated sustained-B firingpattern by setting $g_{\mathrm{KL}}$ to $0.65 \mathrm{mS} / \mathrm{cm}^{2}$. Again, the flow of $I_{\mathrm{KL}}$ and $I_{\mathrm{H}}$ currents during the simulation are shown under the firing pattern (Fig. 7B2,B3). As expected for a model containing $I_{\mathrm{KL}}$ there is some outward current flowing during the resting phase (Fig. 7B2). This translates to a more hyperpolarized resting potential for the sustained-B firing pattern (Fig. $7 B$ ) than for the sustained-A firing pattern (Fig. 7A). During current injection, the spike trains exhibited accommodation as the $I_{\mathrm{KL}}$ current prevented the simulation from reaching threshold, as evidenced by the loss of spikes and emergence of voltage oscillations in the later portions of the current step. In the maximum $I_{\mathrm{H}}$ activation condition, there was much more inward $I_{\mathrm{H}}$ current in the resting condition and a resulting depolarization in resting potential. However, this depolarization shifts the membrane potential further into the activation range for $g_{\mathrm{KL}}$ thereby also increasing the amount of outward flowing $I_{\mathrm{KL}}$. The average outward flowing $I_{\mathrm{KL}}$ is larger in the maximum activation condition than in the minimum activation condition. This illustrates that activation of $I_{\mathrm{H}}$ recruited more $I_{\mathrm{KL}}$ both at rest and during spiking (Fig. 7B2). This recruitment results in spike trains accommodating faster in the maximum-activation condition than in the minimum-activation condition (Fig. 7B1). Thus, in the presence of $I_{\mathrm{KL}}, I_{\mathrm{H}}$ has a net dampening effect on spiking.

\section{The influence of $I_{\mathrm{H}}$ depends on $I_{\mathrm{KL}}$ density and channel composition}

In Figure 7, we showed single examples in which $I_{\mathrm{H}}$ could both excite and dampen spiking, depending on the presence of $I_{\mathrm{KL}}$. In further simulations we varied the size of $g_{\mathrm{KL}}$ from 0 to $0.8 \mathrm{mS} / \mathrm{cm}^{2}$ (sustained-A through transient) while keeping all other conductance densities fixed to explore whether the effect on spiking depends on the relative densities of the two conductances. The composition of $g_{\mathrm{KL}}$ was comprised of one of the following: entirely Kv1 (Fig. 8, green), entirely Kv7 (blue), or equal proportions of the two conductances (magenta). We elicited spike trains using a fixed-length (1500 ms), fixed amplitude (55 pA), suprathreshold current step. Simulations were repeated in both the maximum (solid symbols) and minimum (open symbols) activation conditions for $I_{\mathrm{H}}$ to examine the influence of activating $I_{\mathrm{H}}$. Figure $8 A$ plots the number of spikes evoked by the current step as function of $g_{\mathrm{KL}}$ when comprised entirely by Kv1 type channels. As $g_{\mathrm{KL}}$ increases, the number of spikes evoked by the current step decreases. Beyond a threshold value, there is a dramatic transition, after which spike number drops precipitously with increasing $g_{\mathrm{KL}}$. There are effectively two regimes to the response. First, there is a tonic regime where many spikes are evoked during the current injection and firing patterns are largely sustained-A. Sec- ond, there is a phasic regime where firing patterns are sustained-B and transient.

Because $g_{\mathrm{KL}}$ can be comprised of different channel subtypes, we also examined the influence of channel composition by repeating the above simulations with $100 \% \mathrm{Kv} 7$ or equal proportions of Kv1 and Kv7 (Fig. 8B). Recall that the Kv1 and Kv7 channel models have the same voltage-gated activation range but differ in that Kv1 activates quickly and experiences inactivation whereas the Kv7 activates slowly but does not inactivate (Eqs. $6,7)$. Regardless of channel composition, spike numbers decreased with increasing $g_{\mathrm{KL}}$. However, the amount of $g_{\mathrm{KL}}$ needed to elicit the transition between the tonic and phasic regimes was much smaller when $g_{\mathrm{KL}}$ included Kv7 components. Also, spike number was more sensitive to increases in Kv7 than to increases in Kv1 (seen as a steeper slope in the spike number vs $g_{\mathrm{KL}}$ curve for the Kv7 and mixed conditions). These differences in the effective potency of Kv7 comes from the differences in the net steady-state current produced by the two models; because Kv7 does not inactivate, it produces more steady-state current that the Kv1 model, which experiences significant inactivation. Differences in the time course of activation also impacts firing pattern. Because Kv1 activates much more quickly than Kv7, the transition between the tonic and phasic regimes is much more abrupt for the Kv1 case. In contrast, for both the Kv7 and mixed Kv7/Kv1 conditions, the spike rate (proportional to total number of spikes) slows downs substantially before the transition to the phasic regime.

When $I_{\mathrm{H}}$ is maximally activated, the influence of $g_{\mathrm{KL}}$ is enhanced, and the transition between the tonic and phasic regimes occurs at smaller $g_{\mathrm{KL}}$ values than in the minimum $I_{\mathrm{H}}$ condition (Fig. 8A, $B$, compare dashed lines to dotted lines within each $g_{\mathrm{KL}}$ condition). The influence of shifting the activation of $I_{\mathrm{H}}$ is very different in the regimes before and after the transition. In the tonic regime, the maximum activation condition produces more spikes than the minimum activation condition (i.e., the excitatory effect from the previous figure). However, as $g_{\mathrm{KL}}$ increases, the relative gain in spike numbers provided by $I_{\mathrm{H}}$ diminishes. After the transition, the maximum activation condition produces the opposite effect in that for any fixed value of $g_{\mathrm{KL}}$, there is a decrease in the number of spikes evoked (i.e., the type of dampening seen in Fig. $7 B$ ). Together, these results show that the outward flowing potassium currents carried by $g_{\mathrm{KL}}$ counteract the excitatory influence of $I_{\mathrm{H}}$. Moreover, more $g_{\mathrm{KL}}$ is recruited when the activation range of $I_{\mathrm{H}}$ is depolarized toward the activation range of $g_{\mathrm{KL}}$.

To examine the influence of $I_{\mathrm{H}}$ in isolation from the complexities of $g_{\mathrm{KL}}$ recruitment and kinetics, we plotted the spike number and the regularity of the spike train as a function of $Q_{\mathrm{KL}}$, the net subthreshold charge delivered by $g_{\mathrm{KL}}$ (Fig. $8 C, D$, respectively). We computed $Q_{\mathrm{KL}}$ as the area under the lower envelop of the $I_{\mathrm{KL}}$ versus time curve (Fig. 7B2, hatched area). Note that this is not the same as the net charge delivered by $g_{\mathrm{KL}}$ during the entire spike train, but rather an estimate of the current during the subthreshold portions of the spike-train. As expected, spike number decreases as $Q_{K L}$ increases. Now, however spike number falls along a single curve rather than three parametric curves for each combination of Kv1/Kv7. In this plot, we see that the influence of $I_{\mathrm{H}}$ alone (when isolated from its ability to recruit $I_{\mathrm{KL}}$ ) is excitatory in that it produces more spikes. This is true in both the tonic and phasic regimes (compare open symbols to closed symbols).

To examine the influence of $I_{\mathrm{H}}$ on spike-timing regularity in the simulations, we computed the CV values for the step-evoked spike trains that produced $>10$ spikes (Fig. $8 D$ ); meaning that regularity was only computed within the tonic regime. We make three main observations pertaining to CV. First, all CV values were highly reg- 
A

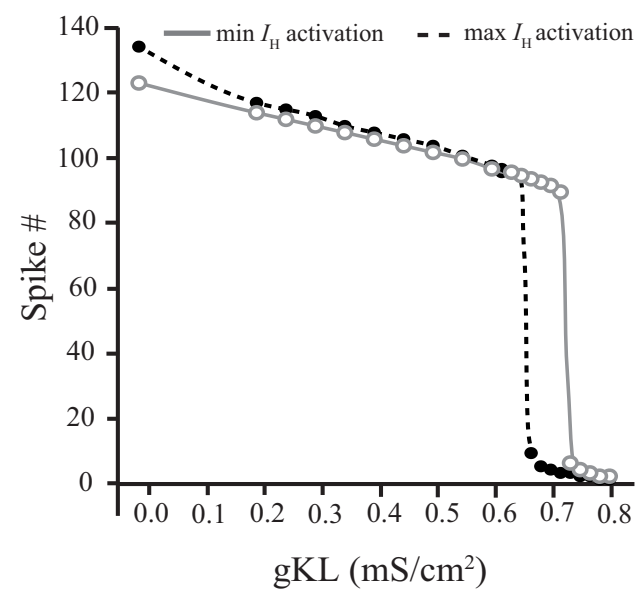

C

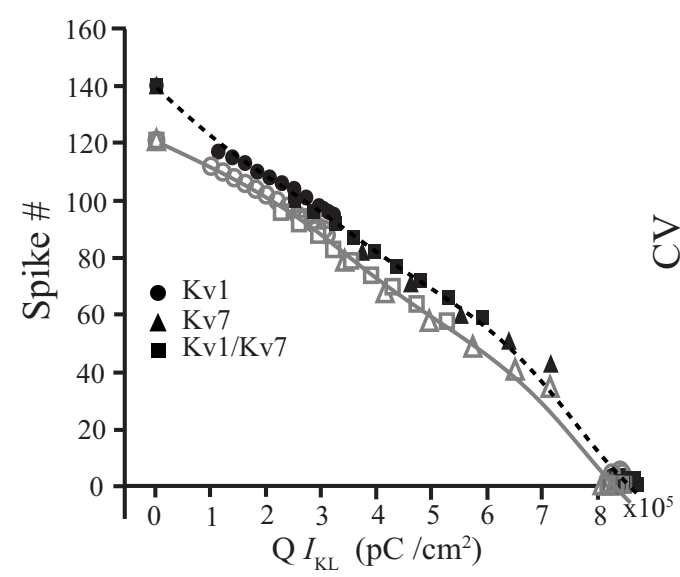

B

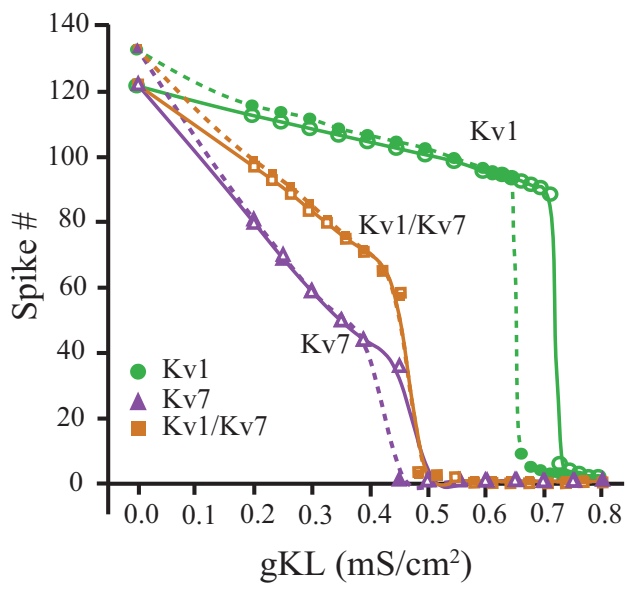

D

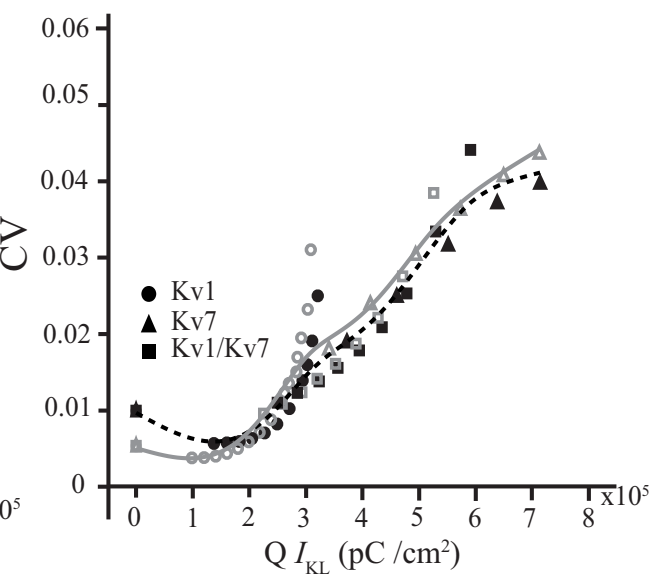

Figure 8. $I_{\mathrm{KL}}$ is the dominant influence on spike number and spike timing. Model VGN with $g_{\mathrm{KL}}$ values between 0.0 and $0.8 \mathrm{mS} / \mathrm{cm}^{2}, \mathrm{Kv} 1$ to $\mathrm{Kv} 7 \mathrm{ratios}$ for $I_{\mathrm{KL}}$ of $1.0 \mathrm{Kv} 1(\mathrm{green}, \bigcirc$ or $\bigcirc$ ), $50 \% \mathrm{Kv} 1$ and Kv7 (magenta, $\square$ or $\square$ ), or $1.0 \mathrm{Kv7}$ (blue, $\mathbf{\Delta}$ or $\Delta$ ) were driven with a $1500 \mathrm{~ms}$ depolarizing current step ( $55 \mathrm{pA}$ ). Each model VGN had either minimum (open symbols) and maximum $I_{H}($ (solid symbols) $I_{\mathrm{H}}$ activation. Trend curves through the data are shown as solid or dashed lines, respectively. $A$, Only spikes during the current step were used to compute spike number. Spike number as a function of $g_{\mathrm{KL}}(1.0 \mathrm{Kv} 1)$ for min and max $I_{\mathrm{H}}$ activation. Spike number falls as a $g_{\mathrm{KL}}$ increases and drops sharply once a large enough $g_{\mathrm{KL}}$ is reached and because of rapid adaption of the spike train (data not shown). Max activation of $/_{\mathrm{H}}$ provides a modest boost in spike number over min activation but requires a smaller $g_{\mathrm{KL}}$ value to illicit adaptation. B, Spike number as a function of $g_{\mathrm{KL}}$ for three model VGNs with $I_{\mathrm{KL}}$ ratios of $1.0 \mathrm{Kv1}, \mathrm{Kv} 1 / \mathrm{Kv} 7$, and $1.0 \mathrm{Kv} 7$. For all three model VGNs, spike number falls as $g_{\mathrm{KL}}$ increases and drops sharply once a large enough $g_{\mathrm{KL}}$ is reached and adaption occurs. This $g_{\mathrm{KL}}$ value is smallest in $1.0 \mathrm{Kv} 7$ and largest in $1.0 \mathrm{Kv} 1 \mathrm{model} V \mathrm{GNs}$. Max activation of $/_{\mathrm{H}}$ condition requires a smaller $g_{\mathrm{KL}}$ value to elicit accommodation for both the $1.0 \mathrm{Kv} 1$ and $1.0 \mathrm{Kv} 7 \mathrm{model} V \mathrm{VGN}$. C, Spike number as a function of net charge carried by $I_{\mathrm{KL}}\left(\mathrm{Q} I_{\mathrm{KL}}, \mathrm{pC} / \mathrm{cm}^{2}\right)$. Activation of $I_{\mathrm{H}}$ provides a modest increase in spike number regardless of net charge by $I_{\mathrm{KL}}$ or composition of $I_{\mathrm{KL}} \cdot D, C \mathrm{C}$ as a function of net charge carried by $I_{\mathrm{KL}}$ for model VGNs that produced at least 10 spikes. $\left(V\right.$ increased as net charge by $I_{\mathrm{KL}}$ increased. CV values overall were highly regular (CV $\left.<0.05\right)$ because of the lack of irregularity imposed by the step stimulus.

ular (CV 0.005-0.05). We attributed this to the stimulus type, compared with the randomly arriving EPSC trains, steps of current impose relatively little irregularity onto the sustained-A model. Second, overall CV increased with $Q_{\mathrm{KL}}$. Because the transition from the tonic to phasic regime was most abrupt for the Kv1 dominated $g_{\mathrm{KL}}$ (Fig. $8 B$ ), the change in regularity was also most abrupt for this channel composition (Fig. 8D, compare green symbols to blue + magenta). Third, activation of $I_{\mathrm{H}}$ produced only modest changes in CV between 2 and $8 \mathrm{pC} / \mathrm{cm}^{2}$ (Fig. 8D, compare solid and dashed trend lines). Spike-timing regularity is much more sensitive to variations in low-voltage-gated potassium currents than to $I_{\mathrm{H}}$. In other words, highly-regular spiking could not be produced by simply increasing $I_{\mathrm{H}}$, but only occurred with small $Q_{\mathrm{KL}}$. Interestingly, $\mathrm{CV}$ depends on $Q_{\mathrm{KL}}$ non-monotonically, with a small upturn toward higher $\mathrm{CV}$ values at the lowest $Q_{\mathrm{KL}}$. In this regime, the step-evoked spike trains are non-adapting with spikes arriving with clock-like precision. Because CV is the ratio of the SD to the mean of spike intervals, the decrease in mean intervals combined with invariance in the SD produces the increase in CV. Similarly, maximizing $I_{\mathrm{H}}$ within this regime produces faster spiking but also produces higher CVs.

\section{Discussion}

A developmental convergence in VGN firing patterns

Here we considered whether highly-regular spiking relies on HCN channels, known to be present in both VGN and calyx terminals (Almanza et al., 2012; Meredith et al., 2012; Horwitz et al., 2014; Yoshimoto et al., 2015). We recorded firing patterns from VGNs after expression for HCN channels is developmentally upregulated (Almanza et al., 2012; Yoshimoto et al., 2015) and we enhanced the activation of $\mathrm{HCN}$-mediated currents (referred to as $I_{\mathrm{H}}$ in the text) by increasing intracellular concentrations of cyclic-AMP (Accili et al., 2002; Almanza et al., 2012; Meredith et al., 2012). With HCN-mediated currents so enhanced, we found faster subthreshold depolarizations and faster firing rates, but did not find more regular spiking. A concomitant 
developmental increase in low-voltage-gated potassium currents (referred to as $I_{\mathrm{KL}}$ in the text) likely countered the influence of $\mathrm{HCN}$-mediated currents to prevent highly-regular spiking.

Ample evidence supports the idea that low-voltage-gated potassium currents grow with development. Patch-clamp recordings in VGNs show that the currents are larger in the second postnatal week than in the first (Kalluri et al., 2010). Consistent with this, we found resting potentials hyperpolarized and input resistance decreased with age. Overall, VGN responses became less diverse, converging toward more phasic and irregular firing patterns.

Developmental upregulation in the Kv7 group of low-voltagegated potassium channels (also known as KCNQ channels) is likely driving this change. Immunohistochemistry and pharmacology showing expression for and regional upregulation of Kv7 channels support this conjecture (Rennie and Streeter, 2006; Pérez et al., 2009; Kalluri et al., 2010; Meredith and Rennie, 2015). Immunolabeling for Kv7 channels is initially confined to neurons innervating the central/striolar zones of the vestibular sensory epithelia and eventually spreads to the peripheral/extrastiolar zones (Hurley et al., 2006). As a consequence, calyx terminals in the peripheral zones have little low-voltage-gated potassium currents during early postnatal days (Songer and Eatock, 2013) but have significant increase in these currents mediated by the second to third postnatal weeks (in gerbils, Meredith and Rennie, 2015; in mice, Ramirez et al., 2018; based on sensitivity to Kv7-specific antagonists, linopiridine or XE991). By P21, nearly all VGNs in mice immunolabel for Kv7 (Rocha-Sanchez et al., 2007). Consistent with this, conversion from transient to sustained spiking can require blocking of both Kv1- and Kv7-mediated low-voltagegated potassium currents (Kalluri et al., 2010). Together, these results suggest that most vestibular afferents eventually acquire low-voltage-gated potassium currents, driven, in part, by a developmental upregulation in Kv7 channels.

\section{Comparison to previous studies}

Iwasaki et al. (2008) described a different developmental trajectory in which a larger fraction of VGNs had tonic/sustained firing patterns as function of development. They interpret this as reflecting an age-dependent increase in HCN channel expression (Yoshimoto et al., 2015) coupled with a relatively age-invariant dependence for $\mathrm{Kvl}$ expression (Iwasaki et al., 2012). This trajectory is satisfying because it describes a pattern of ion channel expression that parallels the in vivo increase in regular-spiking (Curthoys, 1979). However, unlike the present study and those of Soto and colleagues (Limón et al., 2005; Pérez et al., 2009; Almanza et al., 2012), Yoshimoto et al. (2015) recorded from small cells whose size, resting potentials and input resistance did not change with age. Their cells also lacked Kv7-mediated currents because they could convert firing patterns from transient to sustained-firing by only blocking Kv1 channels (Iwasaki et al., 2012). In contrast, we saw a wide range of cells sizes, agedependent hyperpolarization in resting potential and, in a previous study, showed that it is necessary to block both Kv1 and Kv7 channels to convert firing patterns (Kalluri et al., 2010). Whether or not methodological variations, including differences in enzyme composition and maturational sensitivity to enzymes leads to sampling different subpopulation and/or alters ion channel properties remains to be resolved (Quandt, 1987; Matteson and Carmeliet, 1988; Armstrong and Roberts, 1998).

Horwitz et al. (2014) showed impressive control over spiketiming regularity by blocking or enhancing $\mathrm{HCN}$-mediated cur- rents in the calyx terminals of immature dimorphic afferents. This is likely because immature calyces do not yet have their full complement of potassium currents. Our modeling suggests that the absence of low-voltage-gated potassium currents in immature calyces created the ideal condition for HCN-mediated currents to influence spike-timing regularity. Indeed, Songer and Eatock (2013) found sustained spiking and highly regular spontaneous spiking in early postnatal extrastriolar calyxes, indicating that these neurons lacked low-voltage-gated potassium currents. In contrast, striolar calyces have these currents and respond to injected currents with transient/phasic spiking and spontaneously spike with irregular timing. We also considered whether $\mathrm{HCN}$-mediated currents could exert a stronger influence on spike timing at calyx terminals where the current's densities are larger than they are at somata (Horwitz et al., 2014). Our modeling argues against this line of reasoning because even with seven times the HCN channel density found in the somata, the size of low-voltage-gated potassium currents was still the dominant factor controlling spike timing. These results suggest that differences in the size of $\mathrm{HCN}$-mediated currents between terminals and somata do not account for the prevalence of phasic and irregular firing in the present study.

\section{Ion channel mechanisms controlling spike-timing}

$\mathrm{HCN}$-channels are permeable to both sodium and potassium ions, which gives them a reversal potential $\sim-40 \mathrm{mV}$ (which is near spike threshold in VGN; DiFrancesco, 1981; present study). At subthreshold potentials, current flows into the cell through HCN channels. This steady inward current depolarizes the membrane potential toward spike threshold driving highly-regular auto-rhythmic firing (DiFrancesco, 2010; Horwitz et al., 2014). This follows our observations in $500 \mu \mathrm{M} \mathrm{db}$-cAMP, where we found steeper afterhyperpolarization potentials and faster spike rates that are akin to those seen in vivo (Fig. 4). This role of $\mathrm{HCN}$-mediated currents in shaping the afterhyperpolarization potential is similar in spirit (if not in the details) to the phenomenological "afterhyperpolarization current" model for explaining spike-timing regularity in vestibular neurons (Smith and Goldberg, 1986). Because HCN channels inactivate slowly, the resulting steady inward current can raise resting potential and increase net conductance, simultaneously sharpening excitatory postsynaptic potentials and speeding action potential timing (in the auditory nerve, Yi et al., 2010; in the vestibular nerve, Meredith et al., 2012).

$\mathrm{HCN}$-mediated currents influence spiking very differently when they are found with low-voltage-gated potassium currents. As the inward flowing HCN-mediated current depolarizes a neuron, the membrane potential comes closer into the activation range of low-voltage-gated potassium channels. When these potassium channels open, the net outward current works against the $\mathrm{HCN}$-mediated inward current to pull the membrane potential away from spike threshold, making it harder for the neuron to reach spike threshold. How quickly spiking is inhibited depends on the type of low-voltage-gated potassium channel. Fastactivating currents from Kv1 channels quickly tamp-down spiking, whereas the slower activating currents mediated by Kv7 channels often allow several spikes to trigger before the outward current is large enough to squelch spiking (Fig. 7).

The interactions between multiple ion channels determine neuronal excitability, which is a general feature of neuronal biophysics. The role of any particular channel is greatly influenced by the complement of other channels found within neurons' membrane. In early development, HCN channels in vestibular afferents can be ex- 
citatory and drive auto-rhythmic spiking, but, their role flips with the maturational upregulation of potassium channels. This interaction between $\mathrm{HCN}$-mediated and low-voltagegated potassium currents is similar to that described in the auditory brainstem (Bal and Oertel, 2000; Rothman and Manis, 2003; McGinley and Oertel, 2006; Mathews et al., 2010; Cao and Oertel, 2011; Khurana et al., 2012), where HCNmediated currents enhance the inhibitory influence of lowvoltage-gated potassium currents. These results suggest that although HCN-mediated currents grow during maturation, they counter-intuitively suppress VGN excitability by recruiting larger low-voltage-gated potassium currents. This drives maturing VGNs in vitro toward phasic and irregular spiketiming statistics.

If low-voltage-gated potassium currents are ubiquitously upregulated in vestibular afferents, then existing models accounting for spiking-timing regularity need revision. Our results argue against the hypothesis that developmental upregulation in $\mathrm{HCN}$ channel expression is responsible for developmental increases in regular-spiking in vivo. The ion channel complement controlling spike-timing is becoming more complex during maturation. Added complexity comes with added flexibility. In maturing VGN, low-voltage-gated potassium currents are driven through by both Kv1 and Kv7 channels. The Kv7mediated currents can be modulated by cholinergic efferent neurons acting on muscarinic acetylcholine receptors. Muscarinic acetylcholine receptors are a G-protein-coupled receptors that trigger secondary-messenger cascades that ultimately close Kv7 channels (Selyanko et al., 1992; Selyanko and Brown, 1993; for review, see Brown, 2010; for review, see Greene and Hoshi, 2017).

Closing Kv7 channels in VGNs (either directly by blocking the channels or indirectly using the $\mathrm{mAChR}$ agonist oxotremorine-M) decreases membrane conductance, converts in vitro firing patterns from high-threshold transient-firing to low-threshold sustainedfiring (Pérez et al., 2009) and increases the regularity of spontaneous firing in extra-striolar calyces (Ramirez et al., 2018). Consistent with this, turtle vestibular efferent neurons close Kv7 channels to excite a slow increase of in vivo afferent spike rate (Holt et al., 2017). Similarly, blocking Kv7 channels suppresses in vivo afferent response to transient head motions in mice (likely encoded by irregular afferents; Lee et al., 2017). This suggests that maturing vestibular afferents acquire ion channel properties than can be dynamically controlled, with some neurons possibly acquiring the flexibility to move between the regular- and irregular-firing categories, perhaps based on the tone established by descending efferent projections. This is interesting for neurons outside the vestibular system that also require control over their spike timing and suggests one way in which this level of dynamic control can be achieved.

Our results also suggest that temperature differences (e.g., room temperature vs body temperature) do not significantly account for the differences in spike-timing regularity between in vitro and in vivo experiments. We previously found that increasing recording temperature speeds up ion channel kinetics and spike rates (present study; Kalluri et al., 2010). Similarly, spike rates are nearly six times faster at body temperature than room temperature in auditory nerve (Wu et al., 2016). Surprisingly, here we found that in vitro spike-timing for VGNs is neither faster nor more regular at body temperature than at room temperature when cAMP levels are already elevated ( $\mathrm{CV}>0.5$ in both cases). At first blush, this seems surprising because increasing body temperature speeds up ion channel kinetics (shown here for HCN channels in VGNs) and is known to depolarize the voltage activation range of $\mathrm{HCN}$ channels (Gambardella et al., 2012). However, as our experiments show the combination of elevated cAMP concentrations and elevated temperature are not enough to unveil the highly regular spiking found in vivo $(\mathrm{CV}<0.1$; Baird et al., 1988). This supports our primary conclusion that highly regular-spiking in vestibular ganglion neurons is limited by the presence of low-voltage-gated potassium channels and not by the size, speed, and activation state of HCN channels.

Ultimately, the ion channel mechanisms we describe in vitro represent only a piece of the puzzle contributing to in vivo responses. Vestibular afferents have diverse dendritic morphologies, connect to different types and numbers of hair cells in different epithelial zones, and express a variety of ion channels (many were not considered here). In addition to ion channels, models have shown that the size and rate of synaptic events also shapes spike-timing statistics. For example, turtle vestibular neurons having small compact terminal arbors with bouton synapses on few hair cells might produce large and temporally brief synaptic events whereas neurons with extended arbors containing hundreds of bouton-like terminals on many spatially dispersed hair cells may produce small but rapidly arriving events (Goldberg and Holt, 2013; Holmes et al., 2017). A sustained-A model can produce remarkably regular firing patterns when stimulated by a steady depolarization, such as that provided by a step of current (Fig. 8) or by the convergence of small but rapidly arriving synaptic events (Hight and Kalluri, 2016). Consistent with this, regular-firing dimorphic afferents in the peripheral zones of canal afferents appear to have more bouton terminals than those in the central zones (Goldberg et al., 1990b). A similar stimulus with small events arriving at high-rate applied to the transientspiking model cannot even produce a spike train (Hight and Kalluri, 2016). In the future, we will need to consider whether ion channel composition (including efferent modulation) and terminal morphology/connectivity are complimentary specializations, tuned together over development to produce regular and irregular firing patterns.

\section{References}

Accili EA, Proenza C, Baruscotti M, DiFrancesco D (2002) From funny current to HCN channels: 20 years of excitation. News Physiol Sci $17: 32-37$

Almanza A, Luis E, Mercado F, Vega R, Soto E (2012) Molecular identity, ontogeny, and cAMP modulation of the hyperpolarization-activated current in vestibular ganglion neurons. J Neurophysiol 108:2264-2275.

Angelo K, Margrie TW (2011) Population diversity and function of hyperpolarization-activated current in olfactory bulb mitral cells. Sci Rep $1: 50$.

Armstrong CE, Roberts WM (1998) Electrical properties of frog saccular hair cells: distortion by enzymatic dissociation. J Neurosci 18:2962-2973.

Baird RA, Desmadryl G, Fernández C, Goldberg JM (1988) The vestibular nerve of the chinchilla: II. Relation between afferent response properties and peripheral innervation patterns in the semicircular canals. J Neurophysiol 60:182-203.

Bal R, Oertel D (2000) Hyperpolarization-activated, mixed-cation current $\left(I_{\mathrm{h}}\right)$ in octopus cells of the mammalian cochlear nucleus. J Neurophysiol 84:806-817.

Barry PH (1994) JPCalc, a software package for calculating liquid junction potential corrections in patch-clamp, intracellular, epithelial and bilayer measurements and for correcting junction potential measurements. J Neurosci Methods 51: 107-116.

Biel M, Wahl-Schott C, Michalakis S, Zong X (2009) Hyperpolarizationactivated cation channels: from genes to function. Physiol Rev 89:847885 .

Bond CT, Pessia M, Xia XM, Lagrutta A, Kavanaugh MP, Adelman JP (1994) Cloning and expression of a family of inward rectifier potassium channels. Receptors Channels 2:183-191. 
Brown DA (2010) Muscarinic acetylcholine receptors (mAChRs) in the nervous system: some functions and mechanisms. J Mol Neurosci 41:340-346.

Brown HF, DiFrancesco D, Noble SJ (1979) How does adrenaline accelerate the heart? Nature 280:235-236.

Campos-Toimil M, Keravis T, Orallo F, Takeda K, Lugnier C (2008) Shortterm or long-term treatments with a phosphodiesterase-4 (PDE4) inhibitor result in opposing agonist-induced $\mathrm{Ca}^{2+}$ responses in endothelial cells. Br J Pharmacol 154:82-92.

Cao XJ, Oertel D (2011) The magnitudes of hyperpolarization-activated and low-voltage-activated potassium currents co-vary in neurons of the ventral cochlear nucleus. J Neurophysiol 106:630-640.

Chabbert C, Chambard JM, Valmier J, Sans A, Desmadryl G (2001) Hyperpolarization-activated $\left(I_{h}\right)$ current in mouse vestibular primary neurons. Neuroreport 12:2701-2704.

Curthoys IS (1979) The development of function of horizontal semicircular canal primary neurons in the rat. Brain Res 167:41-52.

Desmadryl G, Chambard JM, Valmier J, Sans A (1997) Multiple voltagedependent calcium currents in acutely isolated mouse vestibular neurons. Neuroscience 78:511-522.

DiFrancesco D (1981) A study of the ionic nature of the pace-maker current in calf Purkinje fibres. J Physiol 314:377-393.

DiFrancesco D (2010) The role of the funny current in pacemaker activity. Circ Res 106:434-446.

Felix R, Sandoval A, Sánchez D, Gómora JC, De la Vega-Beltrán JL, Treviño CL, Darszon A (2003) ZD7288 inhibits low-threshold $\mathrm{Ca}^{2+}$ channel activity and regulates sperm function. Biochem Biophys Res Commun 311: 187-192.

Fernández C, Baird RA, Goldberg JM (1988) The vestibular nerve of the chinchilla: I. Peripheral innervation patterns in the horizontal and superior semicircular canals. J Neurophysiol 60:167-181.

Fernández C, Goldberg JM, Baird RA (1990) The vestibular nerve of the chinchilla: III. Peripheral innervation patterns in the utricular macula. J Neurophysiol 63:767-780.

Gambardella C, Pignatelli A, Belluzzi O (2012) The h-current in the substantia nigra pars compacta neurons: a re-examination. PLoS One 7:e52329.

Goldberg JM (2000) Afferent diversity and the organization of central vestibular pathways. Exp Brain Res 130:277-297.

Goldberg JM, Holt JC (2013) Discharge regularity in the turtle posterior crista: comparisons between experiment and theory. J Neurophysiol 110: $2830-2848$

Goldberg JM, Desmadryl G, Baird RA, Fernández C (1990a) The vestibular nerve of the chinchilla: IV. Discharge Properties of Utricular Afferents. J Neurophysiol 63:781-790.

Goldberg JM, Desmadryl G, Baird RA, Fernández C (1990b) The vestibular nerve of the chinchilla: $V$. Relation between afferent discharge properties and peripheral innervation patterns in the utricular macula. J Neurophysiol 63:791-804.

Greene DL, Hoshi N (2017) Modulation of Kv7 channels and excitability in the brain. Cell Mol Life Sci 74:495-508.

Highstein SM, Politoff AL (1978) Relation of interspike baseline activity to the spontaneous discharges of primary afferents from the labyrinth of the toadfish, Opsanus tau. Brain Res 150:182-187.

Hight AE, Kalluri R (2016) A biophysical model examining the role of lowvoltage-activated potassium currents in shaping the responses of vestibular ganglion neurons. J Neurophysiol 116:503-521.

Holmes WR, Huwe JA, Williams B, Rowe MH, Peterson EH (2017) Models of utricular bouton afferents: role of afferent-hair cell connectivity in determining spike train regularity. J Neurophysiol 117:1969-1986.

Holt JC, Jordan PM, Lysakowski A, Shah A, Barsz K, Contini D (2017) Muscarinic acetylcholine receptors and M-currents underlie efferentmediated slow excitation in calyx-bearing vestibular afferents. J Neurosci 37:1873-1887.

Horwitz GC, Risner-Janiczek JR, Holt JR (2014) Mechanotransduction and hyperpolarization-activated currents contribute to spontaneous activity in mouse vestibular ganglion neurons. J Gen Physiol 143:481-497.

Hurley KM, Gaboyard S, Zhong M, Price SD, Wooltorton JR, Lysakowski A, Eatock RA (2006) M-like K+ currents in type I hair cells and calyx afferent endings of the developing rat utricle. J Neurosci 26:10253-10269.

Iwasaki S, Chihara Y, Komuta Y, Ito K, Sahara Y (2008) Low-voltage- activated potassium channels underlie the regulation of intrinsic firing properties of rat vestibular ganglion cells. J Neurophysiol 100:2192-2204.

Iwasaki S, Nakajima T, Chihara Y, Inoue A, Fujimoto C, Yamasoba T (2012) Developmental changes in the expression of $\mathrm{Kvl}$ potassium channels in rat vestibular ganglion cells. Brain Res 1429:29-35.

Kalluri R, Xue J, Eatock RA (2010) Ion channels set spike timing regularity of mammalian vestibular afferent neurons. J Neurophysiol 104:20342051.

Khurana S, Liu Z, Lewis AS, Rosa K, Chetkovich D, Golding NL (2012) An essential role for modulation of hyperpolarization-activated current in the development of binaural temporal precision. J Neurosci 32:2814-2823.

Lee C, Holt JC, Jones TA (2017) Effect of M-current modulation on mammalian vestibular responses to transient head motion. J Neurophysiol 118:2991-3006.

Limón A, Pérez C, Vega R, Soto E (2005) $\mathrm{Ca}^{2+}$-activated K+-current density is correlated with soma size in rat vestibular-afferent neurons in culture. J Neurophysiol 94:3751-3761.

Liu Q, Manis PB, Davis RL (2014) $I_{h}$ and HCN channels in murine spiral ganglion neurons: tonotopic variation, local heterogeneity, and kinetic model. J Assoc Res Otolaryngol 15:585-599.

Lysakowski A, Gaboyard-Niay S, Calin-Jageman I, Chatlani S, Price SD, Eatock RA (2011) Molecular microdomains in a sensory terminal, the vestibular calyx ending. J Neurosci 31:10101-10114.

Mathews PJ, Jercog PE, Rinzel J, Scott LL, Golding NL (2010) Control of submillisecond synaptic timing in binaural coincidence detectors by $\mathrm{K}_{\mathrm{v}} 1$ channels. Nat Neurosci 13:601-609.

Matteson DR, Carmeliet P (1988) Modification of K channel inactivation by papain and $\mathrm{N}$-bromoacetamide. Biophys J 53:641-645.

McGinley MJ, Oertel D (2006) Rate thresholds determine the precision of temporal integration in principal cells of the ventral cochlear nucleus. Hear Res 216-217:52-63.

Meredith FL, Rennie KJ (2015) Zonal variations in K+ currents in vestibular crista calyx terminals. J Neurophysiol 113:264-276.

Meredith FL, Benke TA, Rennie KJ (2012) Hyperpolarization-activated current $\left(\mathrm{I}_{\mathrm{h}}\right)$ in vestibular calyx terminals: characterization and role in shaping postsynaptic events. J Assoc Res Otolaryngol 13:745-758.

Pérez C, Limón A, Vega R, Soto E (2009) The muscarinic inhibition of the potassium $\mathrm{M}$-current modulates the action-potential discharge in the vestibular primary-afferent neurons of the rat. Neuroscience 158:16621674.

Quandt FN (1987) Burst kinetics of sodium channels which lack fast inactivation in mouse neuroblastoma cells. J Physiol 392:563-585.

Ramirez OL, Garrido AG, Koizumi K, Lysakowski A, Eatock RA (2018) KV channels, synaptic transmission and spiking in afferent terminals of the mouse utricular epithelium. 41st Annual Midwinter Meeting for the Association for Research in Otolaryngology. Abs \# Sym30, San Diego.

Rennie KJ, Streeter MA (2006) Voltage-dependent currents in isolated vestibular afferent calyx terminals. J Neurophysiol 95:26-32.

Risner JR, Holt JR (2006) Heterogeneous potassium conductances contribute to the diverse firing properties of postnatal mouse vestibular ganglion neurons. J Neurophysiol 96:2364-2376.

Rocha-Sanchez SM, Morris KA, Kachar B, Nichols D, Fritzsch B, Beisel KW (2007) Developmental expression of Kcnq4 in vestibular neurons and neurosensory epithelia. Brain Res 1139:117-125.

Rothman JS, Manis PB (2003) The roles potassium currents play in regulating the electrical activity of ventral cochlear nucleus neurons. J Neurophysiol 89:3097-3113.

Sadeghi SG, Chacron MJ, Taylor MC, Cullen KE (2007) Neural variability, detection thresholds, and information transmission in the vestibular system. J Neurosci 27:771-781.

Sánchez-Alonso JL, Halliwell JV, Colino A (2008) ZD 7288 inhibits T-type calcium current in rat hippocampal pyramidal cells. Neurosci Lett 439: 275-280.

Schessel DA, Ginzberg R, Highstein SM (1991) Morphophysiology of synaptic transmission between type I hair cells and vestibular primary afferents: an intracellular study employing horseradish peroxidase in the lizard, calotes versicolor. Brain Res 544:1-16.

Selyanko AA, Brown DA (1993) Effects of membrane potential and muscarine on potassium $\mathrm{M}$-channel kinetics in rat sympathetic neurones. J Physiol 472:711-724.

Selyanko AA, Stansfeld CE, Brown DA (1992) Closure of potassium 
M-channels by muscarinic acetylcholine-receptor stimulants requires a diffusible messenger. Proc Biol Sci 250:119-125.

Smith CE, Goldberg JM (1986) A stochastic afterhyperpolarization model of repetitive activity in vestibular afferents. Biol Cybern 54:41-51.

Songer JE, Eatock RA (2013) Tuning and timing in mammalian type I hair cells and calyceal synapses. J Neurosci 33:3706-3724.

Takumi T, Ishii T, Horio Y, Morishige K, Takahashi N, Yamada M, Yamashita T, Kiyama H, Sohmiya K, Nakanishi S, Kurachi Y (1995) A novel ATPdependent inward rectifier potassium channel expressed predominantly in glial cells. J Biol Chem 270:16339-16346.

Wu JS, Young ED, Glowatzki E (2016) Maturation of spontaneous firing properties after hearing onset in rat auditory nerve fibers: spontaneous rates, refractoriness, and interfiber correlations. J Neurosci 36:1058410597.
Wu X, Liao L, Liu X, Luo F, Yang T, Li C (2012) Is ZD7288 a selective block of hyperpolarization-activated cyclic nucleotide-gated channel currents? Channels 6:438-442.

Yamada R, Kuba H, Ishii TM, Ohmori H (2005) Hyperpolarizationactivated cyclic nucleotide-gated cation channels regulate auditory coincidence detection in nucleus laminaris of the chick. J Neurosci 25:8867-8877.

Yi E, Roux I, Glowatzki E (2010) Dendritic HCN channels shape excitatory postsynaptic potentials at the inner hair cell afferent synapse in the mammalian cochlea. J Neurophysiol 103:2532-2543.

Yoshimoto R, Iwasaki S, Takago H, Nakajima T, Sahara Y, Kitamura K (2015) Developmental increase in hyperpolarization-activated current regulates intrinsic firing properties in rat vestibular ganglion cells. Neuroscience 284:632-642. 